\title{
Rational Shaping of Hydrogel by Electrodeposition under Fluid Mechanics for Electrochemical Writing on Complex Shaped Surfaces at Microscale
}

Mariela Alicia Brites Helú ${ }^{1}$ Liang Liu ${ }^{1, *}$

${ }^{1}$ Université de Lorraine, CNRS, Laboratoire de Chimie Physique et Microbiologie pour les Matériaux et l'Environnement (LCPME), F-54000 Nancy, France

*Corresponding author. E-mail: liang.liu@univ-lorraine.fr

\section{Highlights}

- "Electrodeposition + Pulling" is proposed for processing and shaping hydrogel.

- The process synergistically combines electrochemistry with fluid mechanics.

- Rational control relies on electrodeposition kinetics and meniscus evolution.

- Sharp hydrogel probes of width $<1 \mu \mathrm{m}$ are obtained for local electrochemistry.

- Electrochemical writing on complex 3D structures is achieved at tunable resolution. 


\begin{abstract}
The precise control of hydrogel shaping at high resolution is an intrinsic engineering challenge due to the low solid content of the material. Most of the approaches are based on lithographic mold, which becomes tricky to demold when the precision rises to micron and sub-micron range. In this work, a novel automated "electrodeposition + pulling" approach for rationally shaping chitosan hydrogel in $3 \mathrm{D}$ is reported. Taking advantage of the transient meniscus generated while pulling a sharpened metal wire out from a viscous solution, the process confines the electrodeposition of hydrogel simultaneously in it. Considering that the electrodeposition of hydrogel is driven by electrochemically generated $\mathrm{OH}^{-}$ions, the reaction engineering rationally and synergistically combines the mass transport of $\mathrm{OH}^{-}$ions, the gelification and the fluid mechanics in a dynamic process. With automation and mastering the process, the shape of hydrogel can be tuned, and it can be sharpened to below 1 micron at the tip with a desired length. Thus, it is demonstrated for electrochemical writing on 3D printed complex shaped surfaces. The sharpened hydrogel is in soft contact with the sample, allowing electrochemical deposition or etching reactions to be spatially localized in the contact area. By precisely pressing or stretching the hydrogel, the resolution can be tuned from a few to tens of microns just like traditional ink brushes, which may significantly improve the efficiency of writing. The work shows the power of electrochemical reaction engineering towards spatial control in 3D at micro and nano scale.
\end{abstract}

Keywords: Hydrogel, Electrodeposition, Fluid Mechanics, Scanning Probe Microscopy, 3D Printing 


\section{Introduction}

Hydrogel is being widely used as electrolyte in electrochemical energy conversion and storage devices due to its balanced mechanical strength (that serves as support and prevents the leakage) and ionic conductivity [1-4]. It also has excellent biocompatibility, which finds intensive applications as matrix material for stabilizing cells and delivering drugs in bioengineering and biosensing [5-9]. As a solid material, hydrogel shaping is an important issue in its processing. However, due to the softness and sensitivity to physical/chemical stimulus, it is not applicable with traditional machining procedures such as polishing [10]. A classical method for shaping hydrogel is by filling precursor in a mold followed by cross-linking and demolding. However, the demolding step is not easy to control when the resolution rises close to nanoscale due to tearing, fracture and deformation [10], especially when high water content and high precision are both required.

On the other hand, the miniaturization of electrochemical devices calls for gel electrolyte with precise shape control. Consequently, there is also a scientific demand to study the electrochemical reaction kinetics at the gel/electrode interface at micro- and nanoscale. A promising technique is scanning electrochemical cell microscopy (SECCM), where the electrolyte is typically in liquid form localized in a pulled glass capillary. The liquid electrolyte forms a droplet at the opening of the capillary, which bridges the circuit forming a miniaturized electrochemical cell on the sample surface when capillary is approached close to the sample. Preliminary works have also confirmed the feasibility of using hydrogel as electrolyte for SECCM $[11,12]$. The immobilization of liquid electrolyte with gel offers a controlled contact and may inhibit the sample dependent spreading and wetting of electrolyte [13-15]. Nevertheless, in order to improve the control and the spatial resolution, the challenge falls again on the shaping of hydrogel at micro- and nanoscale, which is inherently difficult as the gel naturally tends to be blunt. 
Electrochemistry may also offer a solution for its own need. Electrodeposition of hydrogel is a well-known process based on the local $\mathrm{pH}$ change that induces gelation on the electrode. It is applicable for chitosan, calcium alginate, alkoxysilane, metal alkoxides, etc.[16-18]. From the chemical engineering point of view, electrodeposition offers an additional variable for controlling the process, that is, the potential or current. A few attempts have been made for shaping the hydrogel deposit, such as physically localizing the cathode at microscale or localizing the current distribution by direct mode scanning electrochemical microscopy (SECM), but the precision is mainly in the tens of micron range which is not yet sufficient $[15,19]$. Unfortunately, only electrodeposition is not enough.

Inspired by polymer processing and pulling glass capillaries, in this work we report a novel “electrodeposition + pulling" approach for shaping the electrodeposition of chitosan hydrogel in 3D. By pulling a sharpened metal wire out from a viscous precursor solution, the fluid yields a thin meniscus just like in cheese fondue. Nevertheless, the meniscus is a transient state, and its shape cannot be kept without additional physical or chemical processes such as the evaporation of solvent [20] or crosslinking [21]. Here, taking advantage of this transient state, we carry out electrodeposition at the same time to jellify the meniscus forming sharp chitosan hydrogel. The $\mathrm{pH}$ gradient that triggers chitosan deposition is therefore controlled by both electrolysis of water and meniscus evolution in a dynamic process. Analysis of the physical and chemical effects of the process as well as systematic experimental studies reveal that the key of the "electrodeposition + pulling" process is matching the kinetics of electrodeposition with the dynamics of meniscus evolution, which allows rationally and reproducibly tuning the length, width, and shape of gel deposition for different applications. For example, the sharpened hydrogel is used as tip for direct electrochemical writing on 3D complex shaped surfaces. By precisely controlling the pressing and stretching of gel after contact with the sample, patterns of flexible resolution from a few to tens of microns can be generated either through local electrodeposition or etching. Like in calligraphy and painting with ink brush, large patterns can 
be obtained at relatively low resolution while fine details can still be drawn at high resolution according to the need using the same tip. This is an important feature which may significantly improve the efficiency of writing. All the processes, from the "electrodeposition + pulling" sharpening hydrogel to the electrochemical writing, are fully automated with customized setups and programs. The work demonstrates the power of electrochemical reaction engineering on the spatial control of electrodeposition in 3D, reaching resolution of sub-micron scale for both 3D shaping of hydrogel and the local deposition on 3D printed objects.

\section{Material and methods}

The samples for local surface modification in this work are: Ag foil $(0.125 \mathrm{~mm}$ thick, $99.9 \%$, ChemPur, Germany), $20 \mathrm{~nm}$ thick Ag film sputtered on ITO (8-12 $\Omega$ surface resistivity, Delta Technologies, USA) by a Quorum Q150T sputter coater (Quorum Technologies, UK), and 3D printed model of Eiffel Tower printed on a Pico2 HD printer using SuperCast HD resin (Asiga, Australia) and sputtered with Ag.

Metal wires (Pt, W, Au and $\mathrm{Ag}$ ) are sharpened by electrochemical etching using conditions as listed in SI Section 1.1[22-25]. For "electrodeposition + pulling", the deposition solution consists of 2-2.5 wt.\% chitosan (medium molecular weight, Aldrich), 2-5 wt.\% polyethylene glycol (PEG 2000, Merck), $0.125 \mathrm{~mol} \mathrm{~L}^{-1} \mathrm{NaNO}_{3}$ in 1:1 (vol. ratio) glycerol/ $\mathrm{H}_{2} \mathrm{O}$. PEG has three roles in the process: (1) increases the viscosity of the solution due to higher solid content; (2) reduces the surface tension making the meniscus thinner; (3) enhances the mechanical strength of gel allowing continuous threads of pulling from solution in compensation with the gel/solution interfacial tension. For local chlorination of $\mathrm{Ag}$ surface, $0.125 \mathrm{~mol} \mathrm{~L}^{-1} \mathrm{NaCl}$ is added in the solution. For studying the diffusion behaviour of redox species in the sharpened hydrogel, $5 \mathrm{mmol} \mathrm{L}{ }^{-1} \mathrm{Fc}(\mathrm{MeOH})_{2}$ is added. The $\mathrm{pH}$ is adjusted to around 5.5 using $\mathrm{HNO}_{3}$ or $\mathrm{HCl}$ depending on the anion used in the solution. 
The "electrodeposition + pulling" is carried out on a simple home-built setup consisting of a potentiostat (PalmSens, The Netherlands) and a step motor (Owis, Germany). The sequence is detailed in Fig. 1 and fully automated by a program written in VB.net. All the fabrication parameters $\left(E_{a}, L, E_{h}, t_{h}, E_{w}, v_{w 1}, t_{w 1}, v_{w 2}\right)$ are set in the program and the current is recorded $v s$. time and vertical position $z$.

The local surface modification is also conducted on a home-built setup which is equipped with a potentiostat (PalmSens3, The Netherlands), three step motors in $x, y$ and $z$ axis (Owis, Germany) and a piezo in $z$ axis (PI, Germany). Optical systems (Qioptiq Photonics, UK) coupled with digital cameras (Bresser, Germany) are placed for visualizing the deposition and writing processes for this work, but they are not mandatory. The hardware is controlled by programs written in VB.net. For local electrochemical deposition or etching, the hydrogel probe is approached to be in contact with the conductive surface by current feedback, and then it is further pressed or stretched to a position relative to the touching position where a constant potential is applied for a certain duration. After patterning one point, the hydrogel is rapidly withdrawn to detach from the surface and laterally move to the next desired position, where the approach-electrochemical deposition/etching-withdraw cycle is repeated. The detail experimental parameters are described in the discussion. It should be noted that the local surface modification setup can also be used for "electrodeposition + pulling" sharpening of hydrogel. The morphology of locally modified samples is observed by optical microscope (Nikon, Japan) and further examined by scanning electron microscopy (Zeiss, Germany). The composition is locally analyzed and mapped by EDX (Oxford Instruments, UK). The depth profiles of the patterns are analyzed by profilometer Brüker DetakXT (Brüker, USA) using a $2 \mu \mathrm{m}$ stylus. More details of experimental procedures are given in SI Section 1. The programs for "electrodeposition + pulling" and for local surface modification using hydrogel probes can be shared upon request. 


\section{Results and Discussion}

\subsection{The design of "Electrodeposition + Pulling"}

The novel "electrodeposition + pulling" is a chemical engineering approach that combines the electrochemical generation and mass transport of $\mathrm{OH}^{-}$ions, gelation of chitosan induced by $\mathrm{OH}^{-}$ and fluid mechanics in a dynamic process. Briefly, a sharpened metal wire (of W, Pt, Ag or Au, similar as scanning tunneling microscopy (STM) tips, SI Section 1.1) is immersed in a deposition precursor solution, held for a few seconds and then pulled out, with different potential applied at each step. Due to the viscosity of the solution, a transient meniscus naturally forms during the pulling step before detaching from the solution. We take advantage of this effect and jellify the meniscus by introducing electrodeposition of chitosan at the same time. The synergic use of electrochemistry, mass transport and fluid mechanics makes an elegant approach for shaping hydrogel in 3D.

The process consists of three steps as illustrated in Fig. 1A: (1) Approaching the sharpened metal wire to the deposition solution; (2) Holding the metal wire at a fixed immersion depth while applying potential for inducing electrodeposition; (3) Pulling the metal wire out from the solution while electrodeposition continues by applying potential. The deposition ends when the deposited gel detaches from the solution. The process is fully automated on a customized setup and demonstrated in Video S1 in SI. To rationally tune the shape of gel deposition, we attempt to analyse the process step by step. The readers that are more interested in the experimental control of the process could jump to section 3.2.

\section{Step 1: Approaching}

The approaching step is to approach the cone-shaped etched metal wire to the deposition solution. It is achieved by approaching the wire at a fixed speed $\left(v_{a}\right)$ while applying a potential $\left(E_{a}\right)$. The purpose of $E_{a}$ is to detect the wire-solution contact by current feedback. When the wire touches the solution, a current spike is detected allowing the touching position of the wire 
to be normalized as $z=0$ (Fig. 1B). Once the metal wire touches the solution, the solution "climbs up" along the wire forming a meniscus due to the interfacial tensions. The static shape of the meniscus can be solved from Young-Laplace equation. Due to the axisymmetry of the cone, it is sufficient to solve the curve $R(\theta)$ in polar coordinates with the tip of the etched metal wire cone as origin:

$\gamma_{\text {sol-air }}\left\{\frac{\left[R^{2}+2\left(\frac{d R}{d \theta}\right)^{2}-R\left(\frac{d^{2} R}{d \theta^{2}}\right)\right]\left[\cos \theta \cdot\left(\frac{d R}{d \theta}\right)-R \sin \theta\right]}{\left[R^{2}+\left(\frac{d R}{d \theta}\right)^{2}\right]^{\frac{3}{2}}}+\frac{1+\frac{\tan \theta}{R} \cdot\left(\frac{d R}{d \theta}\right)}{\left[R^{2}+\left(\frac{d R}{d \theta}\right)^{2}\right]^{\frac{1}{2}}}\right\}-\rho_{\text {sol }} g R \sin \theta=0$ (Eq. 1)

The boundary conditions are:

$\left.\frac{d R}{d \theta}\right|_{R=\infty}=\infty(E q .2)$

$\left.\frac{d R}{d \theta}\right|_{\theta=\frac{\pi}{2}-\alpha}=R \frac{\cot (\beta-\alpha) \cos \alpha-\sin \alpha}{\cot (\beta-\alpha) \sin \alpha+\cos \alpha}(E q .3)$

Even without solving the equation, one can see that the solution $R(\theta)$ depends on the half cone angle $(\alpha)$, the density $\left(\rho_{\text {sol }}\right)$ and the surface tension $\left(\gamma_{\text {sol-air }}\right)$ of the deposition solution, as well as the contact angle between the solution and the etched wire $(\beta)$. After touching, the wire is further immersed in the solution by a given length $(L)$. This will affect the solution by changing the pressure term of Eq. 1:

$\gamma_{\text {sol-air }}\left\{\frac{\left[R^{2}+2\left(\frac{d R}{d \theta}\right)^{2}-R\left(\frac{d^{2} R}{d \theta^{2}}\right)\right]\left[\cos \theta \cdot\left(\frac{d R}{d \theta}\right)-R \sin \theta\right]}{\left[R^{2}+\left(\frac{d R}{d \theta}\right)^{2}\right]^{\frac{3}{2}}}+\frac{1+\frac{\tan \theta}{R} \cdot\left(\frac{d R}{d \theta}\right)}{\left[R^{2}+\left(\frac{d R}{d \theta}\right)^{2}\right]^{\frac{1}{2}}}\right\}-\rho_{\operatorname{sol}} g(R \sin \theta-L)=0$ (Eq. 4)

As the etched wire is then held at a fixed position, we can ignore the hydrodynamic motion of solution and consider only the static meniscus $R(\theta)$. This step is only physical as the applied potential $E_{a}$ is not supposed to induce any electrochemical reactions.

\section{Step 2: Holding}

The holding step not only allows the meniscus to reach a steady shape, but also initiates chemical reactions for the deposition of chitosan under an applied potential $E_{h}$ for a duration $t_{h}$. The current response corresponds to the blue segment in Fig. 1B. The mechanism of 
electrodeposition of chitosan is well-known, which is driven by the cathodic generation of $\mathrm{OH}^{-}$ ions from electrolysis of water or electrochemical reduction of dissolved $\mathrm{O}_{2}$ :

$2 \mathrm{H}_{2} \mathrm{O}+2 \mathrm{e} \rightarrow 2 \mathrm{OH}^{-}+\mathrm{H}_{2} \quad(\mathrm{R} 1)$

$\mathrm{O}_{2}+2 \mathrm{H}_{2} \mathrm{O}+4 \mathrm{e} \rightarrow 4 \mathrm{OH}^{-}$

The $\mathrm{OH}^{-}$ions induce the gelation of chitosan by moving the following equilibrium:

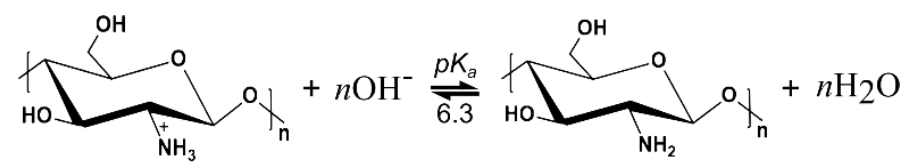

Even though the electrodeposition of chitosan can be well controlled experimentally and has been monitored optically on planar and cylindrical electrodes by Payne et al.[26-28], the quantification of its kinetics is not straightforward. For the ease of treatment, one may look at the deprotonation/protonation of the amino group, which is the driving force of chitosan gelation/dissolution:

$-\mathrm{NH}_{3}{ }^{+}+\mathrm{OH}^{-} \leftrightarrow-\mathrm{NH}_{2}+\mathrm{H}_{2} \mathrm{O}$

Assume the kinetics of R4 is very fast so that the reaction is always at equilibrium, the following relationship is valid:

$K_{e q}=\frac{\left[-\mathrm{NH}_{2}\right]}{\left[-\mathrm{NH}_{3}^{+}\right]\left[\mathrm{OH}^{-}\right]} \quad(\mathrm{Eq} .5)$

where $K_{e q}$ is the equilibrium constant of $\mathrm{R} 4$ which can be easily derived from the $K_{a}$ or $\mathrm{p} K_{a}$ value of chitosan. On the other hand, the solubility product is:

$K_{s p}=\left[-N_{3}^{+}\right]\left[\mathrm{OH}^{-}\right] \quad(E q .6)$

As chitosan is a large molecule and the deposition solution is usually viscous, one may neglect its diffusion and consider that for any $(r, \theta, t)$ the mass balance applies:

$\left[-N H_{3}^{+}\right]+\left[-N H_{2}\right]=C^{*} \quad(E q .7)$

where $C^{*}$ is the initial concentration of amino groups which is constant depending on the concentration of chitosan. Solving Eqs. 5-7, one may derive that the gelation occurs when: 
$\left[O H^{-}\right] \geq \frac{K_{s p}}{C^{*}-K_{s p} K_{e q}} \quad(E q .8)$

Thus, the problem of chitosan film growth turns into the problem of $\mathrm{pH}$ profile. When $\mathrm{pH}$ of the solution rises higher than a threshold, the solution will turn gel deposition. This is consistent with the treatment by Payne et al. where they refer as $\mathrm{pH}$ front matching gel front [29]. It is also in agreement with experimental phenomena where gelation occurs when the $\mathrm{pH}$ of the solution rises higher than 6.3 (the exact value may slightly differ according to the source and molecular weight of chitosan) [30].

At the holding step, the $\mathrm{pH}$ profile can be solved from Fick's second Law with the approximation that $\mathrm{R} 4$ is always at equilibrium (eliminates the kinetic term of the chemical step):

$\frac{\partial\left[\mathrm{OH}^{-}\right]}{\partial t}=D_{\mathrm{OH}^{-}}\left(\frac{\partial^{2}\left[\mathrm{OH}^{-}\right]}{\partial r^{2}}+\frac{1}{r} \frac{\partial\left[\mathrm{OH}^{-}\right]}{\partial r}+\frac{1}{r^{2}} \frac{\partial^{2}\left[\mathrm{OH}^{-}\right]}{\partial \theta^{2}}\right) \quad($ Eq. 9)

The diffusion coefficient of $\mathrm{OH}^{-}$may be different in the gel and the solution:

$D_{O H^{-}}=\left\{\begin{array}{l}D_{s o l}\left(\left[O H^{-}\right]<\frac{K_{s p}}{C^{*}-K_{s p} K_{e q}}\right) \\ D_{g e l}\left(\left[O H^{-}\right] \geq \frac{K_{s p}}{C^{*}-K_{s p} K_{e q}}\right)\end{array}\right.$

The electrode boundary condition is:

$\left.F D_{O H^{-}} \frac{1}{r} \frac{\partial\left[O H^{-}\right]}{\partial \theta}\right|_{\theta=\frac{\pi}{2}-\alpha}=f\left(E,\left[O H^{-}\right]\right)$

where $F$ is the Faraday constant. The function $f\left(E,\left[\mathrm{OH}^{-}\right]\right)$refers to the kinetics of R1 that electrochemically generates $\mathrm{OH}^{-}$ions. Its form depends on the chosen kinetic model (e.g. Butler-Volmer). The surface concentration of other precursors such as $\mathrm{O}_{2}$ or $\mathrm{NO}_{3}{ }^{-}$shall also be taken as variable if other electrochemical reactions than electrolysis of $\mathrm{H}_{2} \mathrm{O}$ that yield $\mathrm{OH}^{-}$are considered. Note that in some literature constant Faradaic current is assumed so that the $\mathrm{OH}^{-}$ flux at the electrode becomes constant regardless of reaction mechanisms for one-dimensional systems. This has advantage for planar, spherical or cylindrical electrodes, yet is not applicable for micro-disk or cone-shaped electrode due to the non-uniform current distribution $[31,32]$. 
Together with the initial condition $\left.\left[\mathrm{OH}^{-}\right]\right|_{t=0}=\left[\mathrm{OH}^{-}\right]_{b u l k}$, the semi-infinite diffusion $\left.\left[\mathrm{OH}^{-}\right]\right|_{r=\infty}=\left[\mathrm{OH}^{-}\right]_{\text {bulk }}$, as well as the no-flux boundary condition at the solution-air interface $r=R(\theta)$, the $\left[\mathrm{OH}^{-}\right]$profile can be solved. The gel is thus formed where $E q .8$ is fulfilled, marking the gel/solution boundary an equal-pH curve of its solution (taking “=”). Note that this analysis does not take into account the possible change of $R(\theta)$ induced by gel formation at the cone electrode. This approximation might not apply in some experimental situations, especially if huge amount of gel is deposited during the holding step (e.g. very cathodic $E_{h}$ for long $t_{h}$, although in practice this should be avoided which will be discussed later).

To have a clue about the diffusion and gel deposition at the holding step, cyclic voltammetry (CV) is carried out on an etched Pt wire when it is placed at $L=20 \mu \mathrm{m}$. Ferrocenedimethanol is added in the solution as redox couple, and its oxidation wave shows a stationary current plateau (Fig. 2A). This indicates that the diffusion is close to radial [33]. By applying a cathodic potential, chitosan can be deposited but mainly at the side of the tip, leaving the very sharp end bare. This is because the spherical diffusion at the tip end is much faster than the radial diffusion at the conical side, so it is more difficult to accumulate sufficient $\mathrm{OH}^{-}$ions to reach the gelation threshold (Eq. 8). The shape of deposition fulfils the diffusion scheme in CV and is also similar to the gradient of redox couple at a conical atomic force microscopy (AFM) probe used for coupling with SECM (AFM-SECM) [34]. It makes the fabrication of sharp gel tip intrinsically difficult by only electrodeposition while holding the etched metal wire at a fixed position, even though the metal wire itself can be extremely sharp having only a few atoms at the tip. On the other hand, the shape in Fig. 2A could well serve as "seed" for the following pulling step which is mandatory for obtaining sharp gel tip.

Step 3: Pulling

After holding, the etched wire is gradually pulled out from the deposition solution at a given speed $\left(v_{w l}\right)$ while applying potential $\left(E_{w}\right)$ allowing electrodeposition to continue. As a consequence, it adds a convection term to Eq. 9 and forms Eq. 12. Unfortunately, even if the 
pulling speed is experimentally controlled in the direction of $\theta=\pi / 2$ (or $z$ axis with cylindrical coordinates that correspond to experimental setup), the velocity term $v$ also depends on the lateral flow induced by the change of the solution-air interface $R(\theta)$. With the gel formation, $R(\theta)$ is no longer the solution of $E q .4$ as the geometry of gel (obviously not a cone anymore, but also not immediately forming cylindrical shape) and interfacial interactions between the gel and the solution shall be considered. The dynamic change of flow also depends on the viscosity of the solution. Due to the complexity, at this stage we do not seek to write the explicit form of the velocity term and solve $E q$. 12. It just helps understanding the process qualitatively.

$\frac{\partial\left[\mathrm{OH}^{-}\right]}{\partial t}=D_{\mathrm{OH}^{-}}\left(\frac{\partial^{2}\left[\mathrm{OH}^{-}\right]}{\partial r^{2}}+\frac{1}{r} \frac{\partial\left[\mathrm{OH}^{-}\right]}{\partial r}+\frac{1}{r^{2}} \frac{\partial^{2}\left[\mathrm{OH}^{-}\right]}{\partial \theta^{2}}\right)+v\left(\frac{\partial\left[\mathrm{OH}^{-}\right]}{\partial r}+\frac{1}{r} \frac{\partial\left[\mathrm{OH}^{-}\right]}{\partial \theta}\right)$ (Eq. 12)

Although the film growth at the initial stage of pulling is no way simple, the termination of film growth at the end of pulling when the gel detaches from the solution could be more clearly analysed with some approximations. During the pulling step, the liquid meniscus shrinks towards $r=0$ and at the same time due to the confined diffusion of $\mathrm{OH}^{-}$it may turn to solid gel. This typically forms cylindrical shaped deposition, which can be regarded as a vertical rod. When its length is much higher than its diameter, one may consider only $1 \mathrm{D}$ diffusion in it (no convection as it moves at the same speed with the wire) by neglecting the curvature of the etched metal wire and treating it approximately as a planar electrode. This is indirectly supported experimentally by measuring $\mathrm{CV}$ of a ferrocenedimethanol labelled sharp hydrogel probe in contact with a platinum plate in a two-electrode system. The voltammogram in Fig. 2B has similar shape as that measured with planar macroelectrodes, indicating that the redox species are likely to follow linear diffusion in the hydrogel. Similar trends were also found in SECCM where the electrolyte is in cylindrical shape in the glass capillary [35].

This 1D diffusion approximation may greatly ease the mathematical treatment and allow an estimation of the evolution of $\mathrm{pH}$ front, or in another word, gel/solution boundary. Eqs. 9-11 change to the following expression in $z$ axis (to be in correspondence to experimental setup): 
$\frac{\partial\left[\mathrm{OH}^{-}\right]}{\partial t}=D_{g e l} \frac{\partial^{2}\left[\mathrm{OH}^{-}\right]}{\partial z^{2}}(\mathrm{Eq} .13)$

$\left.F D_{g e l} \frac{\partial\left[\mathrm{OH}^{-}\right]}{\partial z}\right|_{\mathrm{Z}=0}=f\left(E,\left[O H^{-}\right]\right) \quad(E q .14)$

The gel/solution boundary is the solution of Eq. 8 (taking “=”) and it obviously evolves with time.

As a result, there are three cases for the pulling. Case 1 is that the gel/solution boundary moves faster than the pulling speed. This will induce 3D diffusion and convection of $\mathrm{OH}^{-}$ions in the solution, and likely increase the diameter of the gel by lateral growth. Case 2 is that the gel/solution boundary moves with the same speed as pulling. This should allow the gel to continuously grow in $-z$ direction (or $-\pi / 2$ in polar coordinates) without changing its diameter. However, this is only an ideal situation as the pulling is at a constant speed, but the movement of gel/solution boundary is not, even in 1D diffusion [36]. Case 3 is that the gel/solution boundary moves slower than pulling, which is the most important in this work. It is then like pulling a cylindrical solid object out from a liquid, and the meniscus formed by surface tension may first shrink (decreases diameter) and then detach from the solution. This offers two possible effects: (1) when the shrinking is slow, it may sharpen the gel deposit by further confining the $\mathrm{OH}^{-}$diffusion; (2) when the shrinking is fast, the gel will be rapidly detached from the solution terminating the electrodeposition. Both effects are used in the experimental control. The former is controlled as $v_{w l}$. After certain time $\left(t_{w l}\right)$ when the gel reaches the desired length, it is "cut" by withdrawing at a higher speed $\left(v_{w 2}\right)$, and the deposition terminates when the gel detaches from the solution as indicated by current reducing to zero (with noise in practice, Fig. 1B).

\subsection{Experimental control of "Electrodeposition + Pulling" for shaping gel}

The design above constitutes the basis for rationally controlling the "electrodeposition + pulling" process for shaping gel. Unfortunately, it is already complicated even though we still did not consider many factors such as the friction between phases and the evaporation of 
solvent. More work on the modeling is highly desired, but in this work we focus on the experimental control of the process, which is straightforward and in practice realized with just a button click on our home-built setup.

Even without solving the equations one may see that the process is affected by two competing effects: the gelation driven by electrochemically generated $\mathrm{OH}^{-}$ions, and the meniscus shape change driven by pulling. Both these two effects are linked to deposition parameters in the "electrodeposition + pulling" process. The former can be tuned by deposition potential and time $\left(E_{h}, t_{h}\right.$ and $\left.E_{w}, t_{w 1}\right)$, and the latter is mainly controlled by pulling speed $v_{w 1}$ and $v_{w 2}$. Nevertheless, the two effects are also conjugated in a dynamic process. That is to say, the gelation dynamically changes the gel/solution interface and thereby influences the meniscus, while in reverse the meniscus change also influences the gelation due to the change of boundary. Therefore, the deposition parameters have to be tuned integrally.

The first "taste" of the system is on the kinetics of $\mathrm{OH}^{-}$generation that drive the electrodeposition. This is measured by linear scan voltammetry (LSV) of the etched metal wire in the deposition solution, which helps choosing the potentials $E_{a}, E_{h}$ and $E_{w}$. Fig. 3 shows the LSV of W wire in chitosan solution in cathodic direction. It is seen that the current starts to significantly increase at $c a .-1.1 \mathrm{~V}(v s . \mathrm{Ag} / \mathrm{AgCl} \mathrm{QRE})$, which indicates the onset of water electrolysis that provides $\mathrm{OH}^{-}$ions for driving the jellification of chitosan. Since $E_{a}$ only provides a current feedback to sense the contact of the etched metal wire with the solution, it needs to be more positive ( $\mathrm{ca}$. -0.9 or $-0.8 \mathrm{~V}$ ) so that deposition would not occur while positioning the wire. As a result, $E_{a}$ does not affect the shape of the gel deposition, so it can be fixed without further tuning. In contrary, $E_{h}$ and $E_{w}$ should be selected more negative than the onset potential to induce the electrodeposition of chitosan and their values affect drastically the shape of gel deposition as it will be discussed later.

After approaching the wire at $E_{a}$ reaching the contact with solution, the solution will climb up along the cone due to the capillary effect forming a meniscus that allows electrodeposition on 
the wire. The wire can be further immersed by a distance $L$ to increase the contact area, and the gel deposition is initialized by applying $E_{h}$ for $t_{h}$ as shown in Fig. 1A. This serves as "seed" for the later pulling step. The effect of $E_{h}, t_{h}$ and $L$ is not very sensitive, so the detail optimization is explained in SI (Section 2.1). The main guideline is to induce sufficient deposition and to avoid over deposition.

The core of the "electrodeposition + pulling" is on the pulling step, which tunes the mass transport of $\mathrm{OH}^{-}$ions and orients the electrodeposition by fluid mechanical effect. As discussed in 3.1, the key is to match the pulling speed with the vertical growth rate of gel. As a result, the pulling parameters, namely $E_{w}, v_{w 1}, t_{w 1}$ and $v_{w 2}$, are the most important for tuning the shape of the deposition. Figures 4A-C illustrate the effect of $E_{w}$. At $E_{w}=-1 \mathrm{~V}$ (Fig. 4A), deposition is not observed. This is because the potential is not sufficiently negative to jellify the meniscus so that the liquid goes back to the solution after pulling. By applying a more negative potential $E_{w}$ $=-1.2 \mathrm{~V}$ (Fig. 4B), the deposition is tubular shaped with length of $c a .140 \mu \mathrm{m}$ and width of $c a$. $8 \mu \mathrm{m}$, which may be good enough for doing local electrochemical measurements at high spatial resolution. Applying further negative $E_{w}$ of $-1.4 \mathrm{~V}$ yields longer but much wider deposition (Fig. 4C). This is less favourable for local electrochemical measurements as the spatial resolution is poorer. Therefore, one must control very carefully $E_{w}$ to adjust the kinetics of electrochemical generation of $\mathrm{OH}^{-}$during the pulling process to avoid excess deposition in lateral direction. On the other hand, the mass transport of $\mathrm{OH}^{-}$is affected by the pulling speed. Figures 4D-F show the effect of $v_{w 1}$ in one-step pulling. With slow pulling, the meniscus has enough time to jellify in both lateral and vertical directions, thus the gel grows wider at the root and longer (Fig. 4D). By increasing $v_{w l}$ (Fig. 4E), the meniscus is formed at the same pace with gelation, and therefore yields a thin deposition. Further increasing $v_{w 1}$ would cause rapid detaching of the meniscus, which would make the deposition short (Fig. 4F). Moreover, the gelation is also insufficient due to the short deposition time, making the deposition blunt. The experimental results confirm the high importance of matching the rate of gelation with that of 
pulling for obtaining sharp hydrogel. This is a guideline for fine tuning and balancing $E_{w}$ and $v_{w 1}$ together.

Although the long sharp gel as shown in Fig. 1B, 4B or 4E might serve as micro-capillary reactors for separative sensing [37-39], they may be easily bent and raise issues for certain applications that require good mechanical strength. Therefore, effort is further made for tuning not only the width but also the length of gel deposition in the "electrodeposition + pulling" process. This is achieved by "cutting" the deposition through a second pulling step at a higher speed $v_{w 2}\left(v_{w 2}>v_{w 1}\right)$. With the same deposition conditions as Fig. 4E, Fig. 4G-I shows that the length of gel deposit is significantly reduced by two-step pulling. Moreover, the desired length of the gel $\left(l_{\mathrm{gel}}^{*}\right)$ can be rationally estimated and tuned with acceptable accuracy by setting proper $t_{w 1}:$

$l_{g e l}^{*}=t_{w 1} \cdot r_{g e l}$

where $r_{g e l}$ refers to the gelation rate in vertical direction (parallel to the etched metal wire). The physical meaning of $r_{g e l}$ is the moving speed of the gel/solution boundary, which can be derived from Eqs. 8, 13 and 14 under 1D diffusion approximation. It can be experimentally estimated from the one-step pulling with the same conditions by dividing the length of obtained gel $l_{g e l}$ by the time required for detaching the gel from the solution $t_{\text {detach }}$ :

$r_{\text {gel }}=l_{\text {gel }} /_{t_{\text {detach }}} \quad($ Eq. 16)

For example, from Fig. $4 \mathrm{E}$ it is seen that $l_{\text {gel }}=177 \mu \mathrm{m}$, and $t_{\text {detach }}=127 \mathrm{~s}$ recorded from the current response. Then $r_{g e l}$ is calculated to be $1.4 \mu \mathrm{m} / \mathrm{s}$. Thus, $l_{\text {gel }}^{*}$ is estimated to be 98,49 and $28 \mu \mathrm{m}$ for $t_{w l}=70,35$ and $20 \mathrm{~s}$, respectively. This is generally in accordance with what observed in Fig. 4G-I. Note that the estimation by Eqs. 15 and 16 is empirical, but mathematically not rigorous as $r_{g e l}$ is not a constant but time dependent. It is also observed that the higher the $v_{w 2}$, the more accurate the estimation is. This is because with high speed of pulling at the second step, the deposition would terminate more rapidly after the first pulling. The deposition from 
two-step pulling is slightly wider than that from one-step. This is probably due to less stretching of the gel by the surface tension of the deposition solution. Generally, the aim of the second pulling step is to reduce the length of the gel deposit while keeping the tip sharp. It is beneficial for the applications of these sharp hydrogel in spatially localized electrochemical measurements.

The systematic experimental research above reveals the roles of each deposition parameter in the "electrodeposition + pulling" process, which guides the rational and rigorous tuning of the shape of gel deposition. This is essential for reproducibly tuning and controlling the shape of the sharpened hydrogel towards different applications. For example, tips with different aspect ratio can be fabricated with different deposition parameters (short wide in Fig. 2B or long sharp in Fig. 4B). Practically for optimizing the conditions, the first step is to measure LSV of the metal wire in the deposition solution, which helps fixing $E_{a}$ and estimating the range of $E_{h}, E_{w}$. $L$ slightly depends on the geometry of the etched metal wire and the rheology of the solution, but in most cases, we recommend $L=10-30 \mu \mathrm{m}$. Then, $v_{w l}$ can be optimized together with fine-tuning of $E_{h}, t_{h}$ (recommended to be $<5 \mathrm{~s}$ ) and $E_{w}$ to obtain sharp hydrogel. The last step is to choose $t_{w l}$ according to the desired length of the gel and introduce a second pulling step at $v_{w 2}$ higher than $v_{w 1}$ to terminate the deposition. It should be noted that the exact optimal values of parameters sensitively depend on the deposition precursor, since even slight changes in the composition may affect both the electrodeposition kinetics and the solution properties (surface tension, viscosity, evaporation rate, etc.). Nevertheless, one should not be afraid of adjusting the parameters after customizing a solution (e.g. adding redox couples or functional materials), as the optimizations are rational and not tedious following the general logic, which usually take less than one day to get any desired shape for each new solution. More importantly, once the optimal conditions are settled for a solution, the fabrication is highly reproducible with the automated control (SI section 2.2). 
The "electrodeposition + pulling" process is generic, which applies to various metals such as $\mathrm{W}, \mathrm{Pt}, \mathrm{Ag}$ and $\mathrm{Au}$ (SI Section 1.2). The roles of deposition conditions are similar and sharp hydrogels can always be prepared. In this work, the same $\mathrm{W}$ etched wire is used to get rid of the effect of substrate for systematic studies. After each experiment, the wire is cleaned in acid solution to remove the deposited chitosan and then reused again. Choice of $\mathrm{W}$ is mainly due to its hardness, so that the same etched wire can be used for a long time without significant change of the sharpness of the tip. However, in some cases especially when anodic potential is applied on the gel probe (Fig. 2), Pt is used due to its chemical stability.

\subsection{Application of the shaped gel for electrochemical writing}

Electrochemistry is a powerful technique for modifying surfaces by adding, removing or changing materials (i.e. metals, alloys, polymers, etc.). It can also be spatially localized to generate features from $\mathrm{nm}$ to mm-size by electrochemical writing [40-42], which is achieved typically by scanning electrochemical probe methods such as SECM [43-45] or scanning droplet cell (SDC) $[46,47]$. The early works have been reviewed $[43,48,49]$. State-of-the-art developments allow generation of patterns of nanometer resolution, either by precise control of electrochemical machining on SECM [50,51] or by meniscus-confined deposition $[52,53]$. Structures of 3D are also achieved by vertically lifting the probe [54-56].

The gel probes prepared by "electrodeposition + pulling" may also be used for electrochemical writing. The principle is similar to that of scanning droplet cell, and the lateral resolution of the pattern depends on the gel/sample contact area. This also offers a way to characterize the shape of the probe, as the detail of the sharp tip end cannot be derived from optical images in Fig. 4 due to the round shape and the resolution limit. As illustrated in Fig. 5A, we approach the sharp hydrogel tips to an Ag plate and locally oxidize the contact area while applying an anodic potential bias on Ag. The patterns can be easily tracked by optical/electron microscope due to the high contrast between $\mathrm{AgCl}$ spots and the $\mathrm{Ag}$ surface, and the diameter of spots refers to 
the lateral resolution as reported in our previous work [57]. Furthermore, this model system also allows testing the stability of sharp hydrogel under mechanical stress and demonstrates the feasibility of using "electrodeposition + pulling" fabricated sharp hydrogel probes for electrochemical writing on conductive surfaces, even though the quality of electrodeposited hydrogel may be affected by $\mathrm{Cl}^{-}$ions $[58,59]$.

For writing on surfaces, the first step is to approach the sharp hydrogel probe to be in soft contact with the surface (Fig. 5B). While applying a voltage (e.g. $E_{\text {Sample }}$ vs. $E_{\text {gel }}=0.6 \mathrm{~V}$ ) between the sample and the hydrogel probe (two-electrode system), the current is initially zero (with noise) when the probe is far away from the sample (Segment a, $z<<0$ ). Once the probe touches the sample, the current shows a spike (Segment b) which provides a feedback for normalizing the touching position as $z=0$. The probe could further be pressed or retracted to the desired $z$ position (Segment $\mathrm{c}$, here showing pressing $z>0$ ) like in calligraphy. After positioning, the probe is held for a desired period under applied voltage for writing. The current gradually decreases, perhaps due to the formation of $\mathrm{AgCl}$ that passives the $\mathrm{Ag}$ surface (Segment d). After writing, the hydrogel probe is rapidly retracted from the sample (Segment e). Current is still detected when the probe goes up beyond the touching point $(z<0)$. Similar phenomenon has been observed in our previous work using other gel electrodes [60], and it is because the gel could be stretched to counterbalance its adhesion to the sample. Once the stretching force exceeds the adhesion, the gel is fully detached from the sample and the current returns to zero with noise (Segment $f, z<<0$ ). Then the probe is laterally moved to the next writing point, and the approaching-holding-retracting cycle is repeated, as illustrated in Fig. 5A. It should be noted that the approaching, holding, and retracting is more often carried out at different potentials to reduce the undesired surface change during the approaching and retracting steps. Fig. 5C shows a patterned array of 256 spots generated at $E_{\text {Sample }} v s$. $E_{\text {gel }}=0.7$ $\mathrm{V}$ with a hydrogel probe of low aspect ratio (Fig. 5D). For each spot, the hydrogel probe is positioned at $z=1 \mu \mathrm{m}$ (approaching and retracting at $E_{\text {Sample }} v s$. $E_{g e l}=0.4 \mathrm{~V}$ ) and held for $8 \mathrm{~s}$. 
From SEM images, it is seen that the patterned spots are highly homogeneous, consisting of $\mathrm{AgCl}$ particles as confirmed by EDX mapping (Fig. S7). The size of the patterned spots is $c a$. $6 \mu \mathrm{m}$ (Fig. 5D) and the shape is not perfectly round. Nevertheless, the size and shape of the spots become highly reproducible after the first two rows ( $c a .32$ spots). The first two rows with slightly bigger size of spots are an artifact, likely because the fresh hydrogel contains free absorbed water that may spread on the sample surface upon contact. Similar phenomenon was also observed on approach-retract curves in our previous work [60], so we recommend executing a few approach-retract cycles before starting serious writing (similar as removing excess liquid in brush painting). Following the same writing sequence, other large patterns are made (see, SI section 2.3). Fig. 5D summarizes the morphological characteristics of single $\mathrm{AgCl}$ spots taken from them, along with the hydrogel probes used for writing. The size of the spots varies from tens $(>60 \mu \mathrm{m})$ to just few microns $(1-2 \mu \mathrm{m})$ according to the sharpness of the employed hydrogel probe. Since the size of $\mathrm{AgCl}$ spots is determined by the contact area between the sharpened hydrogel and the Ag surface, it reflects the diameter of the gel probe and indicates the physical lateral resolution ( $R$; the pixel size) of electrochemical writing. It is seen that "Electrodeposition + Pulling" is able to provide hydrogel probes in a wide range of resolution, possibly as high as $c a .0 .5 \mu \mathrm{m}$.

It is important to note that different resolutions can be achieved not only by using hydrogel probes of different size as showed in Fig. 5D but also by stretching and pressing the same hydrogel probe. As the hydrogel is elastic, it could be repeatedly stretched or pressed in a range without losing its original shape. This offers flexible resolution in the range of tens of microns for the same gel probe as demonstrated in SI Fig. S9. In practice, this improves the efficiency of writing since insensitive areas of a pattern can be generated with lower resolution by pressing the hydrogel to reduce the number of pixels, while fine details can still be drawn at higher resolution by stretching the same gel probe. The electrochemical writing is also demonstrated 
for etching $\mathrm{Ag}$ films at different resolution using $\mathrm{NO}_{3}{ }^{-}$-containing hydrogel probes (SI, section 2.4).

Overall, the hydrogel probes prepared by "electrodeposition + pulling" offer a reliable and tunable lateral resolution of less than $10 \mu \mathrm{m}$. This is higher than commercial ink-jet printing and most of the electrochemical patterning work by SECM [43-45,61] or fluid force microscopy [62], yet still has a gap with optical/ion/electron beam-based lithography [63-65] or dip pen lithography [66,67]. Nevertheless, the conventional local surface modification techniques are mainly applicable only to highly flat surfaces such as silicon wafer, whereas "writing" on complex shaped surfaces remains a technical challenge. As demonstrated, the resolution of the hydrogel probes is tunable in the range of microns, which matches that of 3D printed structures $(c a .100 \mu \mathrm{m})$. Therefore, it has a potential useful application for the local generation of functionalities on $3 \mathrm{D}$ printed structures as a post-treatment. The feasibility of using hydrogel probes prepared by "electrodeposition + pulling" for local electrochemical surface modification of complex shape is first examined by purposely tilting the sample surface (SI section 2.5). It is found that the size of written spots does not change when the sample is tilted up to $45^{\circ}$. The gel probes can even write on more steep surfaces with tilt up to $10^{\circ}$.

The sharp hydrogel probes prepared by "electrodeposition + pulling" are further challenged for writing at the sharp edge of a surgical blade, as shown in Fig. 6A. Spots of $c a .5 \mu \mathrm{m}$ in diameter are written precisely over the edge (dashed line), having each of their halves at both sides of the sharp edge while the overall circular shape of the spots is maintained. Fig. 6B illustrates the electrochemical writing on a 3D-printed model using the hydrogel probes made by "electrodeposition + pulling". The sample is simply placed "as printed" without any special adjustment of positioning direction (SI, Video S2). The pattern consists of two parallel rows of spots written across a channel with a step height of $200 \mu \mathrm{m}$ (SI, Video S3). The spots are written at $z=+5 \mu \mathrm{m}$ by applying $E_{\text {Sample }} v s$. $E_{g e l}=1.2 \mathrm{~V}$ for $30 \mathrm{~s}$ for each spot. They are almost the same in size and shape, even though they cannot be all in the same focus under optical 
microscope. More examples of writing on the side detail and sharply curved edges of the same model are shown in SI (section 2.6). These patterns in Fig. 6 (and SI) demonstrate the applicability of electrochemical writing on extreme surfaces in terms of the complexity of the sample geometry, using the gel probes accurately fabricated by "electrodeposition + pulling". The immobilization of electrolyte in the form of soft but stable gel prevents the leakage and spreading of liquid over the sample surface, which is especially important for edges, steps or steep surfaces [13]. Important engineering aspects such as the speed of writing and the stability of the gel are also discussed in SI Section 3.

\section{Conclusions}

We report a novel "electrodeposition + pulling" method for tuning the shape of electrodeposition of chitosan hydrogel in 3D. The beauty relies on the synergy of electrochemical deposition with fluid mechanics, which allows the dynamic process to be rationally and reproducibly controlled for obtaining gel deposition of desired shape. The effect of deposition parameters is discussed from the design aspect and systematically studied experimentally, and it is revealed that the key to the process is the matching of rate for the electrochemically induced gelation and the evolution of meniscus. The "electrodeposition + pulling" method is generic for different metals (W, Pt, $\mathrm{Ag}$ and $\mathrm{Au}$ ), and is foreseen to be applicable to any hydrogel that can be electrodeposited. The process is in practice fully automated just like pulling glass capillaries, offering sharp hydrogels that are used for electrochemical writing on 3D printed complex shaped surfaces, e.g. sharp edges, steps, and steep side planes. The hydrogel probes are positioned to be in contact with the sample by current feedback, and local modification of the sample surface is carried out by electrochemical deposition or etching. The lateral resolution reaches up to $c a$. 1-2 $\mu \mathrm{m}$, and more importantly it is tuneable by pressing or stretching the same hydrogel due to the soft nature of the gel. With the resolution matching that of $3 \mathrm{D}$ printing, the hydrogel probe offers a promising tool for post- 
treatment of $3 \mathrm{D}$ printed structures to generate local functionalities on the surface. Note that local electrochemical modification of surfaces is just one application of such sharpened hydrogel probes prepared by "electrodeposition + pulling". By introducing more sophisticated (bio)chemistry to functionalize the hydrogel, one may foresee various applications such as sensing and high-resolution multi-signal (bio)electrochemical imaging in future [68].

\section{Acknowledgments}

The authors gratefully acknowledge financial support from CNRS MOMENTUM Project (2018-2020) and State Key Laboratory of Physical Chemistry of Solid Surfaces, Xiamen University. The platform of Spectroscopies and Microscopies of Interfaces (SMI) of LCPME and Dr. Mathieu Etienne are also acknowledged for providing part of the hardware platform.

\section{Declarations}

\section{Funding}

This work was funded by MOMENTUM Project (2018-2020) of Centre National de la Recherche Scientifique and State Key Laboratory of Physical Chemistry of Solid Surfaces, Xiamen University.

\section{Conflicts of interest/Competing interests}

There are no conflicts to declare.

\section{Availability of data and material}

The authors confirm that the data supporting the findings of this study are available within the article and its supplementary materials.

\section{Code availability}

The programs for "electrodeposition + pulling" and for local surface modification using hydrogel probes can be shared upon request. 


\section{References}

[1] Z. Wang, H. Li, Z. Tang, Z. Liu, Z. Ruan, L. Ma, Q. Yang, D. Wang, C. Zhi, Hydrogel Electrolytes for Flexible Aqueous Energy Storage Devices, Adv. Funct. Mater. 28 (2018) 1-30. https://doi.org/10.1002/adfm.201804560.

[2] J. Zhao, J. Gong, G. Wang, K. Zhu, K. Ye, J. Yan, D. Cao, A self-healing hydrogel electrolyte for flexible solid-state supercapacitors, Chem. Eng. J. 401 (2020) 125456. https://doi.org/10.1016/j.cej.2020.125456.

[3] R. Na, Y. Liu, N. Lu, S. Zhang, F. Liu, G. Wang, Mechanically robust hydrophobic association hydrogel electrolyte with efficient ionic transport for flexible supercapacitors, Chem. Eng. J. 374 (2019) 738-747. https://doi.org/10.1016/j.cej.2019.06.004.

[4] W. Yang, X. Wang, R. Rossi, B.E. Logan, Low-cost Fe-N-C catalyst derived from Fe (III)-chitosan hydrogel to enhance power production in microbial fuel cells, Chem. Eng. J. 380 (2020) 122522. https://doi.org/10.1016/j.cej.2019.122522.

[5] A. Jayakumar, V.K. Jose, J.M. Lee, Hydrogels for Medical and Environmental Applications, Small Methods. 4 (2020) 1-19. https://doi.org/10.1002/smtd.201900735.

[6] R.A. Siegel, Y. Gu, M. Lei, A. Baldi, E.E. Nuxoll, B. Ziaie, Hard and soft micro- and nanofabrication: An integrated approach to hydrogel-based biosensing and drug delivery, J. Control. Release. 141 (2010) 303-313. https://doi.org/10.1016/j.jconrel.2009.12.012.

[7] Y. Chen, Y. Qiu, Q. Wang, D. Li, T. Hussain, H. Ke, Q. Wei, Mussel-inspired sandwichlike nanofibers/hydrogel composite with super adhesive, sustained drug release and anti$\begin{array}{lllllll}\text { infection capacity, } & \text { Chem. } & \text { Eng. J. } 399 & \text { (2020) } & & 39668 .\end{array}$ https://doi.org/10.1016/j.cej.2020.125668.

[8] J. Wu, G. Li, T. Ye, G. Lu, R. Li, L. Deng, L. Wang, M. Cai, W. Cui, Stem cell-laden injectable hydrogel microspheres for cancellous bone regeneration, Chem. Eng. J. 393 (2020) 124715. https://doi.org/10.1016/j.cej.2020.124715.

[9] M.S. Baktash, A. Zarrabi, E. Avazverdi, N.M. Reis, Development and optimization of a new hybrid chitosan-grafted graphene oxide/magnetic nanoparticle system for $\begin{array}{llllll}\text { theranostic applications, J. Mol. } & \text { Liq. } & \text { (2020) }\end{array}$ https://doi.org/10.1016/j.molliq.2020.114515. 
[10] M.A. Rose, J.J. Bowen, S.A. Morin, Emergent Soft Lithographic Tools for the Fabrication of Functional Polymeric Microstructures, ChemPhysChem. 20 (2019) 909925. https://doi.org/10.1002/cphc.201801140.

[11] H. Kang, S. Hwang, J. Kwak, A hydrogel pen for electrochemical reaction and its applications for 3D printing, Nanoscale. $7 \quad$ (2010) 994-1001. https://doi.org/10.1039/c4nr06041e.

[12] S. Pei, M. Kang, P. Wilson, L. Meng, D. Perry, A. Basile, P.R. Unwin, High resolution visualization of the redox activity of $\mathrm{Li} 2 \mathrm{O} 2$ in non-aqueous media: conformal layer vs. toroid structure, Chem. Commun. $54 \quad$ (2018) 3053-3056. https://doi.org/10.1039/c7cc09957f.

[13] D. Thatenhorst, J. Rheinlaender, T.E. Schäffer, I.D. Dietzel, P. Happel, Effect of sample slope on image formation in scanning ion conductance microscopy, Anal. Chem. 86 (2014) 9838-9845. https://doi.org/10.1021/ac5024414.

[14] N. Dang, M. Etienne, A. Walcarius, L. Liu, Scanning gel electrochemical microscopy (SGECM): The potentiometric measurements, Electrochem. Commun. 97 (2018) 64-67. https://doi.org/10.1016/j.elecom.2018.10.020.

[15] L. Liu, M. Etienne, A. Walcarius, Scanning Gel Electrochemical Microscopy for Topography and Electrochemical Imaging, Anal. Chem. 90 (2018) 8889-8895. https://doi.org/10.1021/acs.analchem.8b01011.

[16] E.R. Cross, The electrochemical fabrication of hydrogels: a short review, SN Appl. Sci. 2 (2020) 397. https://doi.org/10.1007/s42452-020-2194-5.

[17] K. Ino, F. Ozawa, N. Dang, K. Hiramoto, S. Hino, R. Akasaka, Y. Nashimoto, H. Shiku, Biofabrication Using Electrochemical Devices and Systems, Adv. Biosyst. 4 (2020) 115. https://doi.org/10.1002/adbi.201900234.

[18] J. Li, S. Wu, E. Kim, K. Yan, H. Liu, C. Liu, H. Dong, X. Qu, X. Shi, J. Shen, W.E. Bentley, G.F. Payne, Electrobiofabrication: electrically based fabrication with biologically derived materials, Biofabrication. $11 \quad$ (2019) 0-31. https://doi.org/10.1002/adbi.201900234.

[19] T. Danieli, D. Mandler, Local surface patterning by chitosan-stabilized gold 
nanoparticles using the direct mode of scanning electrochemical microscopy (SECM), J. Solid State Electrochem. 17 (2013) 2989-2997. https://doi.org/10.1007/s10008-0132194-0.

[20] D. Zhan, D. Yang, B.-S. Yin, J. Zhang, Z.-Q. Tian, Electrochemical Behaviors of Single Microcrystals of Iron Hexacyanides/NaCl Solid Solution, Anal. Chem. 84 (2012) 92769281. https://doi.org/10.1021/ac302053x.

[21] K. Hou, H. Wang, Y. Lin, S. Chen, S. Yang, Y. Cheng, B.S. Hsiao, M. Zhu, Large Scale Production of Continuous Hydrogel Fibers with Anisotropic Swelling Behavior by Dynamic-Crosslinking-Spinning, Macromol. Rapid Commun. 37 (2016) 1795-1801. https://doi.org/10.1002/marc.201600430.

[22] C. Zhang, B. Gao, L.G. Chen, Q.S. Meng, H. Yang, R. Zhang, X. Tao, H.Y. Gao, Y. Liao, Z.C. Dong, Fabrication of silver tips for scanning tunneling microscope induced luminescence, Rev. Sci. Instrum. 82 (2011) 083101-1-083101-5. https://doi.org/10.1063/1.3617456.

[23] M. Lopes, T. Toury, M.L. De La Chapelle, F. Bonaccorso, P. Giuseppe Gucciardi, Fast and reliable fabrication of gold tips with sub-50 $\mathrm{nm}$ radius of curvature for tip-enhanced Raman spectroscopy, Rev. Sci. Instrum. 84 (2013) 073702-1-073702-8. https://doi.org/10.1063/1.4812365.

[24] G. Tahmasebipour, Y. Hojjat, V. Ahmadi, A. Abdullah, Effect of fabrication process parameters on the apex-radius of STM tungsten nanotip, Scanning. 31 (2009) 65-74. https://doi.org/10.1002/sca.20142.

[25] L. Libioulle, Y. Houbion, J.M. Gilles, Very sharp platinum tips for scanning tunneling microscopy, Rev. Sci. Instrum. 66 (1995) 97-100. https://doi.org/10.1063/1.1146153.

[26] K. Yan, C. Yang, W. Zhong, Z. Lu, X. Li, X. Shi, D. Wang, Wire templated electrodeposition of vessel-like structured chitosan hydrogel by using a pulsed electrical signal, Soft Matter. 16 (2020) 9471. https://doi.org/10.1039/d0sm01134g.

[27] Y. Cheng, X. Luo, J. Betz, S. Buckhout-White, O. Bekdash, G.F. Payne, W.E. Bentley, G.W. Rubloff, In situ quantitative visualization and characterization of chitosan electrodeposition with paired sidewall electrodes, Soft Matter. 6 (2010) 3177-3183. 
https://doi.org/10.1039/c0sm00124d.

[28] S. Wu, K. Yan, J. Li, R.N. Huynh, C.B. Raub, J. Shen, X. Shi, G.F. Payne, Electrical cuing of chitosan's mesoscale organization, React. Funct. Polym. 148 (2020) 104492. https://doi.org/10.1016/j.reactfunctpolym.2020.104492.

[29] K. Yan, F. Ding, W.E. Bentley, H. Deng, Y. Du, G.F. Payne, X.-W. Shi, Coding for hydrogel organization through signal guided self-assembly, Soft Matter. 10 (2014) 465469. https://doi.org/10.1039/c3sm52405a.

[30] V.Zargar, M. Asghari, A. Dashti, A Review on Chitin and Chitosan Polymers: Structure, Chemistry, Solubility, Derivatives, and Applications, ChemBioEng Rev. 2 (2015) 204226. https://doi.org/10.1002/cben.201400025.

[31] A.J. Bard, L.R. Faulkner, Electrochemical Methods: Fundamentals and Applications, 2nd ed., New York, 2008.

[32] S. Yaeli, E. Binyamin, S. Shoham, Form-function relations in cone-tipped stimulating $\begin{array}{lllll}\text { microelectrodes, } & \text { Front. } & \text { Neuroeng. } & 2 & \text { (2009) }\end{array}$ https://doi.org/10.3389/neuro.16.013.2009.

[33] A. V. Sokirko, K.B. Oldham, The voltammetric response of a conical electrode, J. Electroanal. Chem. 430 (1997) 15-24. https://doi.org/10.1016/S0022-0728(96)04909-1.

[34] C. Kranz, G. Friedbacher, B. Mizaikoff, A. Lugstein, J. Smoliner, E. Bertagnolli, Integrating an Ultramicroelectrode in an AFM Cantilever: Combined Technology for Enhanced Information, Anal. Chem. 73 (2001) 2491-2500. https://doi.org/10.1021/ac001099v.

[35] M.E. Snowden, A.G. Güell, S.C.S. Lai, K. McKelvey, N. Ebejer, M.A. Oconnell, A.W. Colburn, P.R. Unwin, Scanning electrochemical cell microscopy: Theory and experiment for quantitative high resolution spatially-resolved voltammetry and simultaneous ion-conductance measurements, Anal. Chem. 84 (2012) 2483-2491. https://doi.org/10.1021/ac203195h.

[36] L. Liu, A. Walcarius, Kinetics of the electrochemically-assisted deposition of sol-gel films, Phys. Chem. Chem. Phys. $19 \quad$ (2017) 14972-14983. https://doi.org/10.1039/c7cp01775h. 
[37] A.I. Barbosa, N.M. Reis, A critical insight into the development pipeline of microfluidic immunoassay devices for the sensitive quantitation of protein biomarkers at the point of care, Analyst. 142 (2017) 858-882. https://doi.org/10.1039/c6an02445a.

[38] J. Pivetal, F.M. Pereira, A.I. Barbosa, A.P. Castanheira, N.M. Reis, A.D. Edwards, Covalent immobilisation of antibodies in Teflon-FEP microfluidic devices for the sensitive quantification of clinically relevant protein biomarkers, Analyst. 142 (2017) 959. https://doi.org/10.1039/c6an02622b.

[39] A.I. Barbosa, J.H. Wichers, A. van Amerongen, N.M. Reis, Towards One-Step Quantitation of Prostate-Specific Antigen (PSA) in Microfluidic Devices: Feasibility of Optical Detection with Nanoparticle Labels, Bionanoscience. 7 (2017) 718-726. https://doi.org/10.1007/s12668-016-0390-y.

[40] H. Liu, S. Hoeppener, U.S. Schubert, Nanoscale Materials Patterning by Local Electrochemical Lithography, Adv. Eng. Mater. $18 \quad$ (2016) 890-902. https://doi.org/10.1002/adem.201500486.

[41] A.D. Davydov, V.M. Volgin, Electrochemical Local Maskless Micro/Nanoscale Deposition, Dissolution, and Oxidation of Metals and Semiconductors (A Review), Russ. J. Electrochem. 56 (2020) 52-81. https://doi.org/10.1134/S1023193520010036.

[42] S. Kee, P. Zhang, J. Travas-Sejdic, Direct writing of 3D conjugated polymer micro/nanostructures for organic electronics and bioelectronics, Polym. Chem. 11 (2020) 4530. https://doi.org/10.1039/d0py00719f.

[43] A.J. Bard, M. V. Mirkin, eds., Scanning electrochemical microscopy, 2nd ed., CRC Press, New York, 2012.

[44] L. Liu, C. Tan, J. Chai, S. Wu, A. Radko, H. Zhang, D. Mandler, Electrochemically “writing" graphene from graphene oxide, Small. 10 (2014) 3555-3559. https://doi.org/10.1002/smll.201301953.

[45] M.A. Brites Helú, J.L. Fernández, Strategies for fabrication of highly-dense ordered arrays of metal microdisks by the scanning electrochemical microscopy microwriting approach, Sensors Actuators B. $250 \quad$ (2017) 420-428. https://doi.org/10.1016/j.snb.2017.04.155. 
[46] E.E. Oseland, Z.J. Ayres, A. Basile, D.M. Haddleton, P. Wilson, P.R. Unwin, Surface patterning of polyacrylamide gel using scanning electrochemical cell microscopy (SECCM), Chem. Commun. 52 (2016) 9929-9932. https://doi.org/10.1039/c6cc05153g.

[47] J. Clausmeyer, J. Henig, W. Schuhmann, N. Plumeré, Scanning droplet cell for chemoselective patterning through local electroactivation of protected quinone monolayers, $\quad$ ChemPhysChem. $\quad 15 \quad$ (2014) 151-156. https://doi.org/10.1002/cphc.201300937.

[48] R. Maoz, S.R. Cohen, J. Sagiv, Nanoelectrochemical Patterning of Monolayer Surfaces: Toward Spatially Defined Self-Assembly of Nanostructures, Adv. Mater. 11 (1999) 5561. https://doi.org/10.1002/(SICI)1521-4095(199901)11:1<55::AIDADMA55>3.0.CO;2-8.

[49] S. Sarkar, D. Mandler, Scanning Electrochemical Microscopy versus Scanning Ion Conductance Microscopy for Surface Patterning, ChemElectroChem. 4 (2017) 29812988. https://doi.org/10.1002/celc.201700719.

[50] L. Han, Z. Hu, M.M. Sartin, X. Wang, X. Zhao, Y. Cao, Y. Yan, D. Zhan, Z.Q. Tian, Direct Nanomachining on Semiconductor Wafer By Scanning Electrochemical Microscopy, Angew. Chemie - Int. Ed. 59 (2020) 21129-21134. https://doi.org/10.1002/anie.202008697.

[51] L. Han, M.M. Sartin, Z.-Q. Tian, D. Zhan, Z.-W. Tian, Electrochemical nanomachining, Curr. Opin. Electrochem. 22 (2020) 80-86. https://doi.org/10.1016/j.coelec.2020.05.007.

[52] K. McKelvey, M.A. O’Connell, P.R. Unwin, Meniscus confined fabrication of multidimensional conducting polymer nanostructures with scanning electrochemical cell microscopy (SECCM), Chem. Commun. $49 \quad$ (2013) 2986-2988. https://doi.org/10.1039/c3cc00104k.

[53] Y. Lei, X. Zhang, D. Xu, M. Yu, Z. Yi, Z. Li, A. Sun, G. Xu, P. Cui, J. Guo, D. Guggenheim, Dynamic “Scanning-Mode” Meniscus Confined Electrodepositing and Micropatterning of Individually Addressable Ultraconductive Copper Line Arrays, J. Phys. Chem. Lett. 9 (2018) 37. https://doi.org/10.1021/acs.jpclett.8b00636.

[54] G. Ercolano, C. van Nisselroy, T. Merle, J. Vörös, D. Momotenko, W.W. Koelmans, T. 
Zambelli, Additive manufacturing of sub-micron to sub-mm Metal structures with hollow AFM cantilevers, Micromachines. $11 \quad$ (2020) 1-14. https://doi.org/10.3390/mi11010006.

[55] J. Hu, M.F. Yu, Meniscus-confined three-dimensional electrodeposition for direct writing of wire bonds, Science. $329 \quad$ (2010) 313-316. https://doi.org/10.1126/science.1190496.

[56] S. Morsali, S. Daryadel, Z. Zhou, A. Behroozfar, D. Qian, M. Minary-Jolandan, Multiphysics simulation of metal printing at micro/nanoscale using meniscus-confined electrodeposition: Effect of environmental humidity, J. Appl. Phys. 121 (2017). https://doi.org/10.1063/1.4973622.

[57] N. Dang, M. Etienne, A. Walcarius, L. Liu, Scanning Gel Electrochemical Microscopy (SGECM): Lateral Physical Resolution by Current and Shear Force Feedback, 92 (2020) 6415-6422. https://doi.org/10.1021/acs.analchem.9b05538.

[58] C.C. Tsai, B.H. Morrow, W. Chen, G.F. Payne, J. Shen, Toward Understanding the Environmental Control of Hydrogel Film Properties: How Salt Modulates the Flexibility of Chitosan Chains, Macromolecules. 50 (2017) 5946-5952. https://doi.org/10.1021/acs.macromol.7b01116.

[59] Y. Liu, B. Zhang, K.M. Gray, Y. Cheng, E. Kim, G.W. Rubloff, W.E. Bentley, Q. Wang, G.F. Payne, Electrodeposition of a weak polyelectrolyte hydrogel: remarkable effects of salt on kinetics, structure and properties, Soft Matter. 9 (2013) 2703-2710. https://doi.org/10.1039/c3sm27581g.

[60] L. Liu, M. Etienne, A. Walcarius, SI- Scanning Gel Electrochemical Microscopy for Topography and Electrochemical Imaging, Anal. Chem. 90 (2018) 8889-8895. https://doi.org/10.1021/acs.analchem.8b01011.

[61] J. Zhang, J. Jia, L. Han, Y. Yuan, Z.-Q. Tian, Z.-W. Tian, D. Zhan, Kinetic Investigation on the Confined Etching System of n-Type Gallium Arsenide by Scanning Electrochemical Microscopy, J. Phys. Chem. C. 118 (2014) 18604-18611. https://doi.org/10.1021/jp5056446.

[62] L. Hirt, R.R. Grüter, T. Berthelot, R. Cornut, J. Vörös, T. Zambelli, Local surface 
modification via confined electrochemical deposition with FluidFM $\uparrow$, RSC Adv. 5 (2015) 84517-84522. https://doi.org/10.1039/c5ra07239e.

[63] T. Ito, S. Okazaki, Pushing the limits of lithography, Nature. 406 (2000) 1027-1031. https://doi.org/10.1038/35023233.

[64] B.D. Gates, Q. Xu, M. Stewart, D. Ryan, C.G. Willson, G.M. Whitesides, New approaches to nanofabrication: Molding, printing, and other techniques, Chem. Rev. 105 (2005) 1171-1196. https://doi.org/10.1021/cr030076o.

[65] S. Kitayaporn, J.H. Hoo, K.F. Böhringer, F. Baneyx, D.T. Schwartz, Orchestrated structure evolution: Accelerating direct-write nanomanufacturing by combining topdown patterning with bottom-up growth, Nanotechnology. 21 (2010) 195306. https://doi.org/10.1088/0957-4484/21/19/195306.

[66] G. Liu, S.H. Petrosko, Z. Zheng, C.A. Mirkin, Evolution of Dip-Pen Nanolithography (DPN): From Molecular Patterning to Materials Discovery, Chem. Rev. 120 (2020) 6009-6047. https://doi.org/10.1021/acs.chemrev.9b00725.

[67] W. Shim, A.B. Braunschweig, X. Liao, J. Chai, J.K. Lim, G. Zheng, C.A. Mirkin, Hardtip, soft-spring lithography, Nature. $469 \quad$ (2011) 516-521. https://doi.org/10.1038/nature09697.

[68] A. Kisieliute, A. Popov, R.M. Apetrei, G. Cârâc, I. Morkvenaite-Vilkonciene, A. Ramanaviciene, A. Ramanavicius, Towards microbial biofuel cells: Improvement of charge transfer by self-modification of microoganisms with conducting polymer Polypyrrole, Chem. Eng. J. $356 \quad$ (2019) 1014-1021. https://doi.org/10.1016/j.cej.2018.09.026. 


\section{Table and Figure Captions}

Fig. 1 A- Scheme of the fabrication of sharpened hydrogel by "electrodeposition + pulling", BTypical current response during the fabrication, Inset: Photo of the obtained sharp hydrogel.

Fig. $2 \mathrm{~A}-\mathrm{CV}$ of $5 \mathrm{mM} \mathrm{Fc}(\mathrm{MeOH})_{2}$ at a Pt sharpened wire held at $L=20 \mu \mathrm{m}$, scheme of radial diffusion of $\mathrm{OH}^{-}$and photo of the obtained gel deposition. B- $\mathrm{CV}$ of $5 \mathrm{mM} \mathrm{Fc}(\mathrm{MeOH})_{2}$ at a Pthydrogel probe, scheme of lineal diffusion of $\mathrm{OH}^{-}$within the meniscus and photo of hydrogel probe prepared by "Electropedosition + Pullling".

Fig. 3 LSV at a W sharpened wire on chitosan deposition solution.

Fig. 4 Influence of "Electrodeposition + Pulling" parameters during the pulling step. Conditions of deposition: $E_{a}=-0.7 \mathrm{~V} ; L=20 \mu \mathrm{m} ; E_{h}=-1.2 \mathrm{~V} ; t_{h}=5 \mathrm{~s}$. Scale bars: $25 \mu \mathrm{m}$.

Fig. 5 A-Scheme of local modification of (non)planar Ag surfaces using hydrogel probes. BTypical current response during pattering one spot. C- SEM image of an array of $\mathrm{AgCl}$ spots on $\mathrm{Ag}$ plate. D- SEM images of individual $\mathrm{AgCl}$ spots and photos of the used hydrogel probes (scale bar: $25 \mu \mathrm{m}$ ).

Fig. 6 A- (I) and (II) Photo and scheme of a gel probe approaching to the surgical blade; (III) $\mathrm{AgCl}$ spots on the very edge of a surgical blade. B- (I) 3D printed Eiffel Tower model coated with Ag; (II) Scheme of the locally modified area; (III) $\mathrm{AgCl}$ spots patterned on the area shown in (II). 

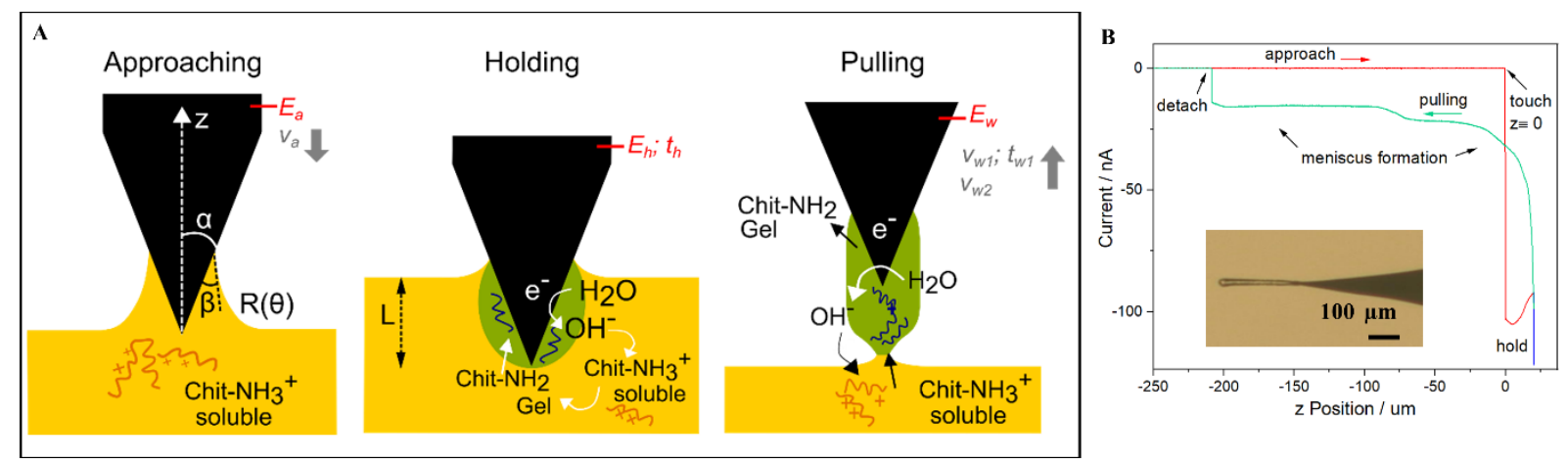

Fig. 1.

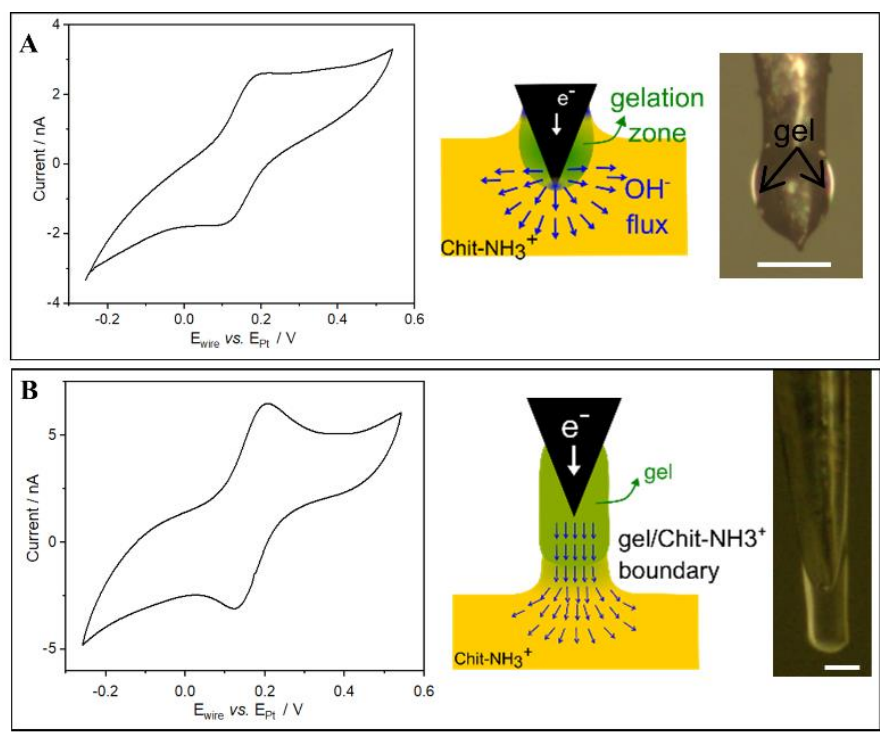

Fig. 2.

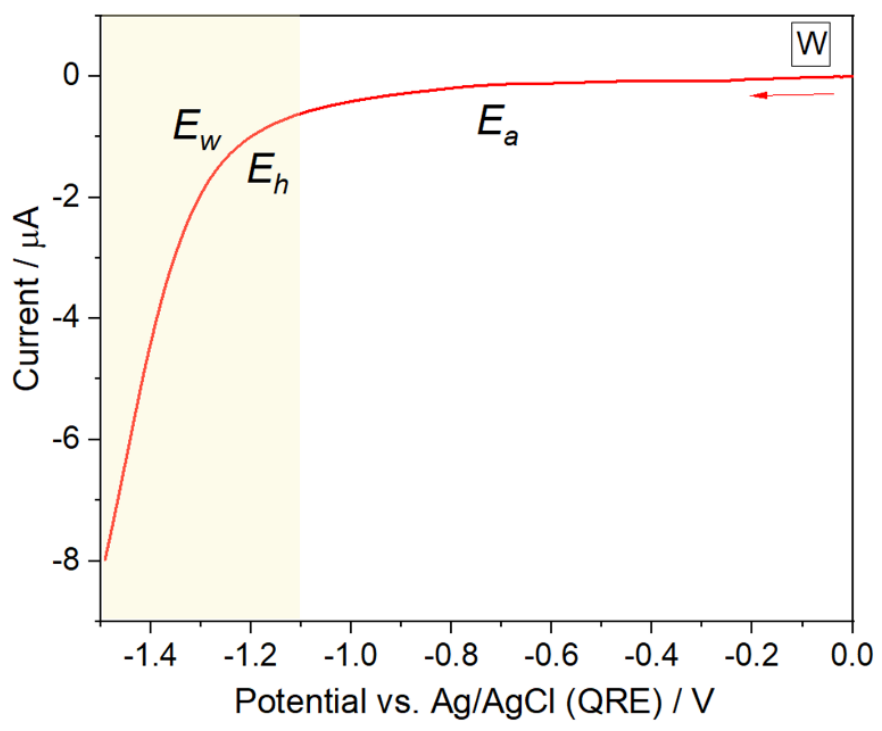

Fig. 3. 


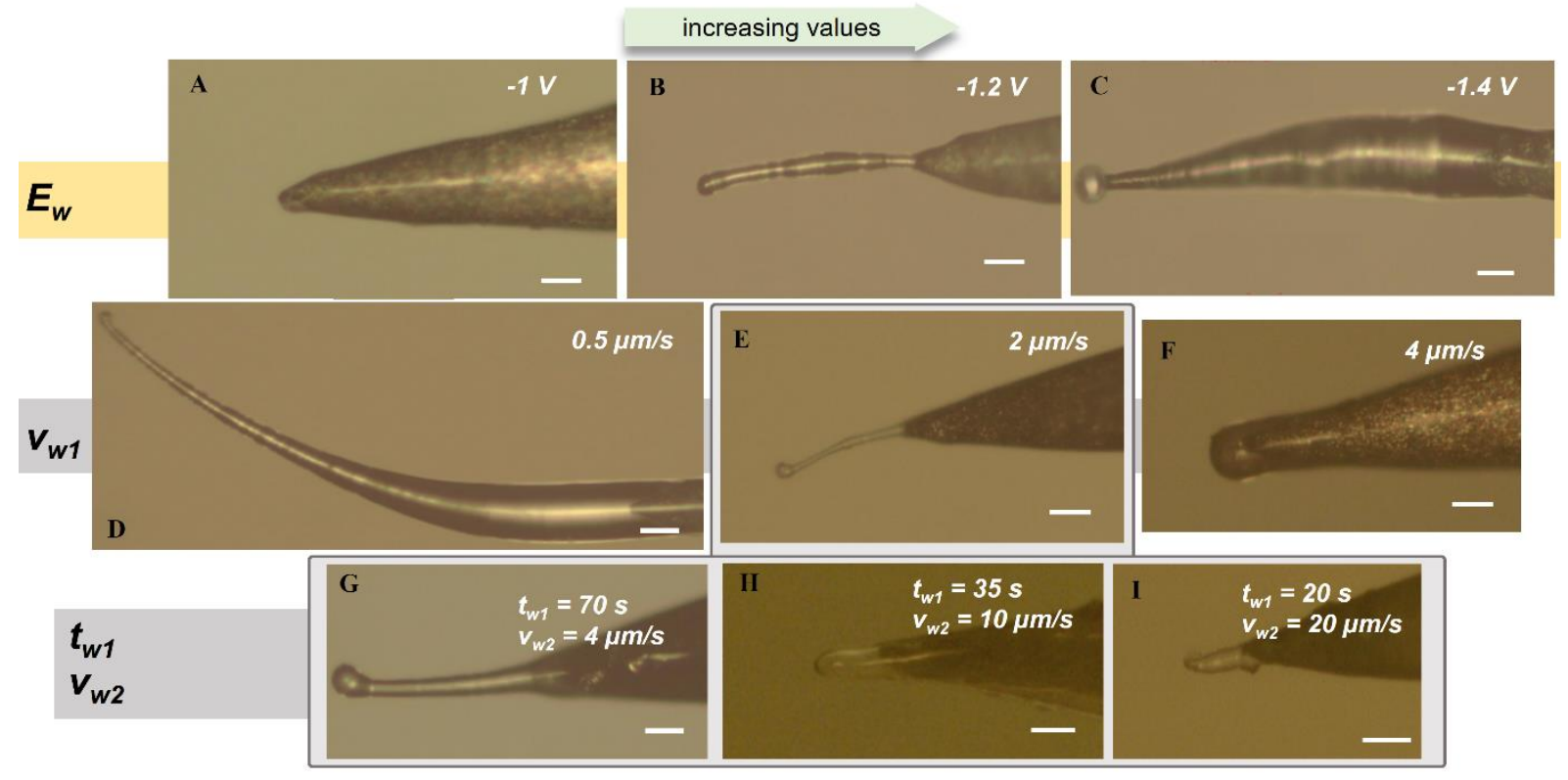

Fig. 4.
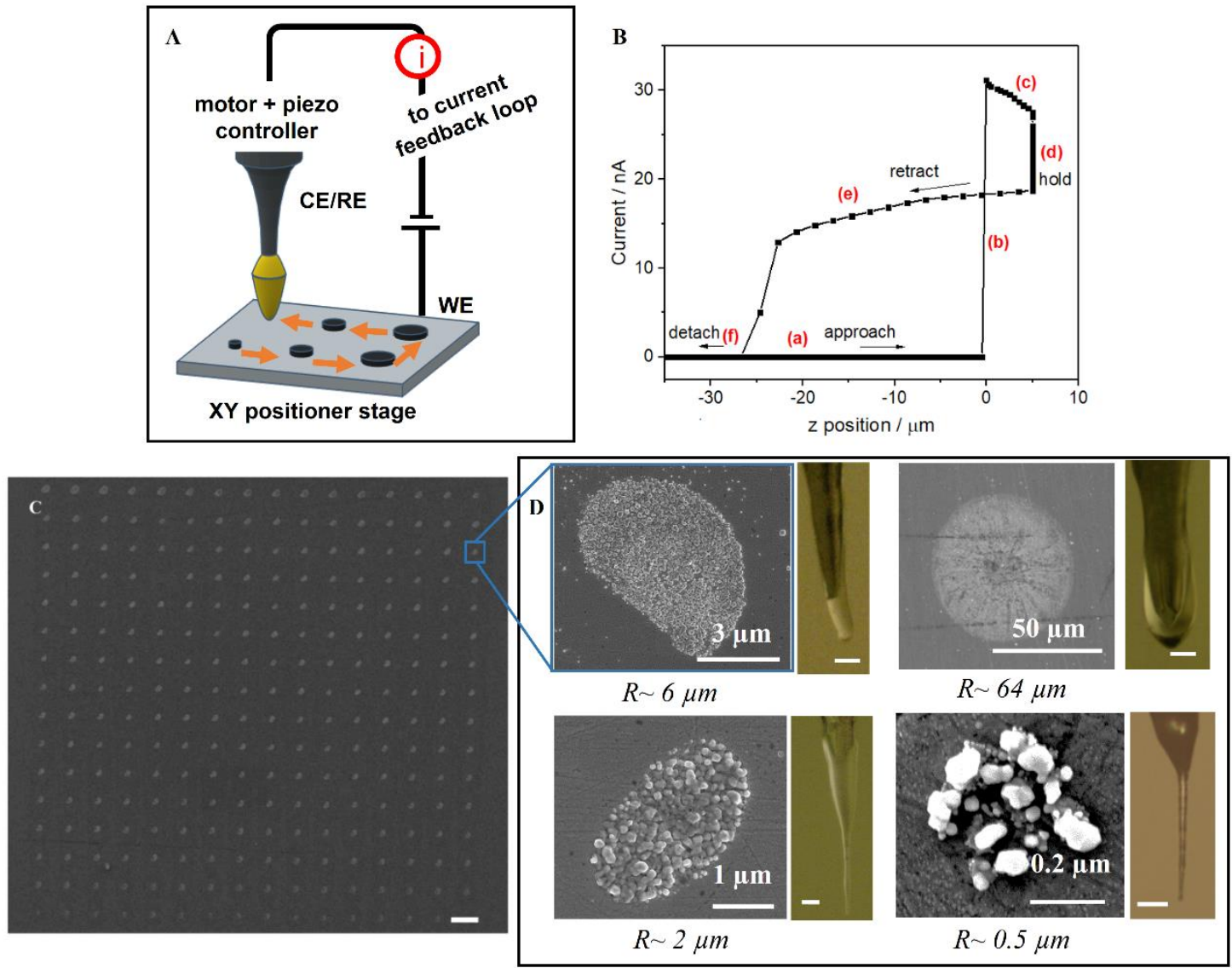

Fig. 5. 


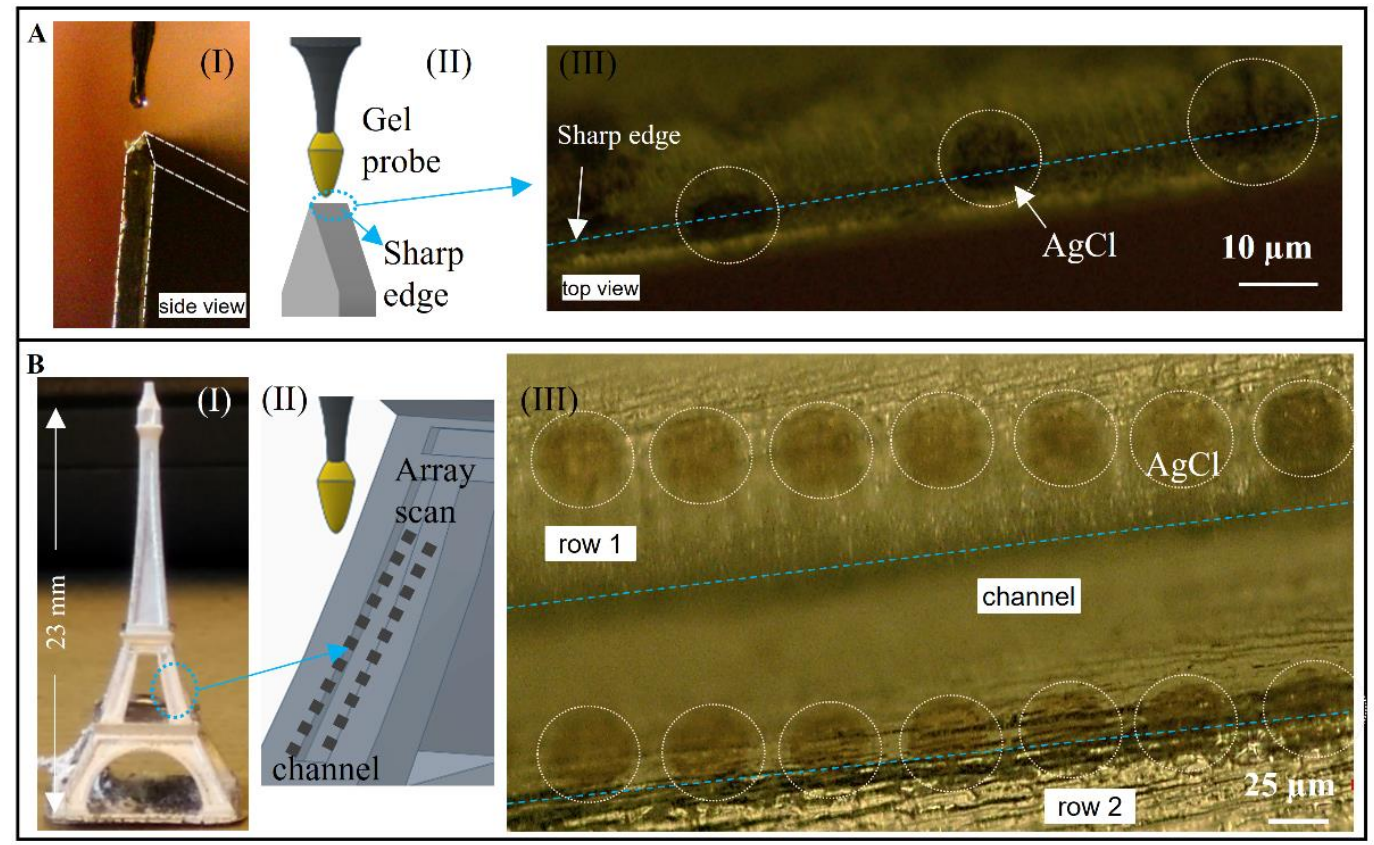

Fig. 6. 
Supporting Information for

\section{Rational Shaping of Hydrogel by Electrodeposition under Fluid Mechanics for Electrochemical Writing on Complex Shaped Surfaces at Microscale}

Mariela Alicia Brites Helú ${ }^{1}$, Liang Liu ${ }^{1, *}$

${ }^{1}$ Université de Lorraine, CNRS, Laboratoire de Chimie Physique et Microbiologie pour les Matériaux et l'Environnement (LCPME), F-54000 Nancy, France

*Corresponding author. E-mail: liang.liu@univ-lorraine.fr

\section{Table of Contents}

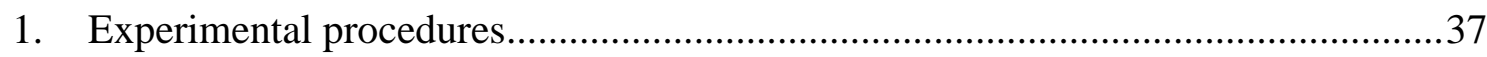

1.1. Preparation of sharpened metal wires by electrochemical etching ...................37

1.2. "Electrodeposition + Pulling" of chitosan on etched Pt, Au and Ag wires ......37

1.3. Experimental setup for local electrochemical modification of a 3D printed model 38

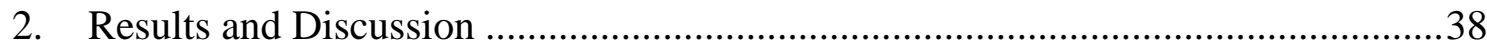

2.1. Influence of holding parameters on the shape of the hydrogel........................38

2.2. Reproducibility of "electrodeposition + pulling" ...........................................4

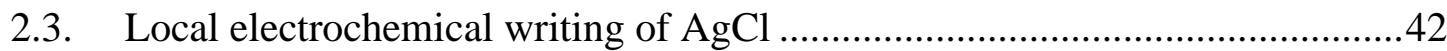

2.4. Local electrochemical etching of $\mathrm{Ag}$.............................................................4

2.5. Effect of sample tilt on the electrochemical writing.......................................45

2.6. Local electrochemical modification of a 3D printed Eiffel Tower....................46

3. Technical aspects: printing speed and stability of hydrogel probes........................47

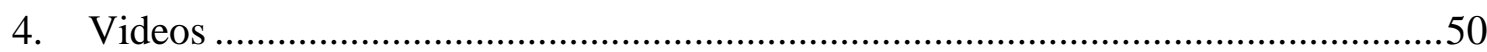

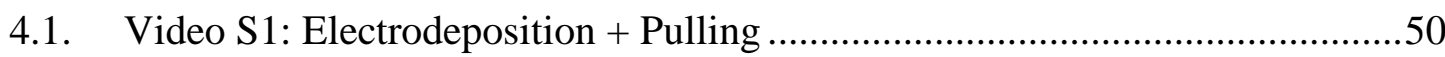

4.2. Video S2: Local modification of 3D printed Eiffel Tower model ....................51

4.3. Video S3: Writing at the step edge of the 3D printed Eiffel Tower ..................51

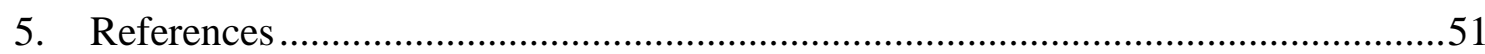




\section{Experimental procedures}

\subsection{Preparation of sharpened metal wires by electrochemical etching}

Metal wires of $\mathrm{W}, \mathrm{Pt}, \mathrm{Au}$ and $\mathrm{Ag}$ are sharpened following similar protocols for preparing scanning tunnelling microscope (STM) probes[1-4]. The metal wires are immersed in the electrolyte by ca. 1 $\mathrm{mm}$ in a two-electrode setup with a ring counter electrode (Fig. S1). Then a DC or AC voltage is applied between the wire and the counter electrode. The wire is etched to cone shape until being detached from the solution. Table S1 lists the conditions for etching different metals. The etched wires are cleaned by sonication in ethanol before depositing chitosan. The unetched part of the wire is sealed in glass capillary with epoxy for the convenience of holding it.

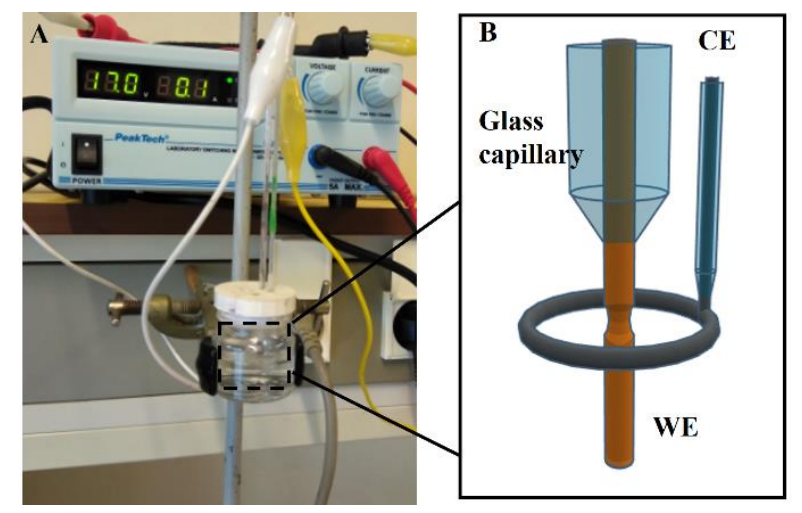

Fig. S1. A- Photo of the experimental setup for electrochemical etching of metal wires. B- Scheme of the two-electrode configuration for etching.

Table S1. Conditions employed in the preparation of sharpened metal wires.

\begin{tabular}{|c|c|c|c|}
\hline Metal & Solution & Voltage & Average time of etching \\
\hline W & $5 \mathrm{M} \mathrm{NaOH}$ & $17 \vee D C$ & $50 \mathrm{~s}$ \\
\hline Pt & $\begin{array}{c}1.5 \mathrm{M} \mathrm{CaCl}_{2} \text { in } 1: 1 \\
\mathrm{H}_{2} \mathrm{O} / \text { Acetone (vol. } \\
\text { ratio) }\end{array}$ & $15 \mathrm{VAC}$ & $2 \min$ \\
\hline $\mathrm{Au}$ & $\mathrm{HCl}$ conc. & $3 \vee D C$ & $2 \min$ \\
\hline $\mathrm{Ag}$ & $\begin{array}{l}\mathrm{MeOH} / \mathrm{HClO}_{4} \text { 1:4 } \\
\text { (vol. ratio) }\end{array}$ & $3 \vee D C$ & $30 \mathrm{~s}$ \\
\hline
\end{tabular}

\section{2. "Electrodeposition + Pulling" of chitosan on etched Pt, Au and Ag wires}

Besides $\mathrm{W}$ as shown in the main text, other metals such as Pt, Au and Ag could also be etched forming sharp tips that are used for "electrodeposition + pulling" of chitosan. The examples of 
depositions are shown in Fig. S2. This proves the versatility of the "electrodeposition + pulling" approach for fabricating sharp hydrogel.
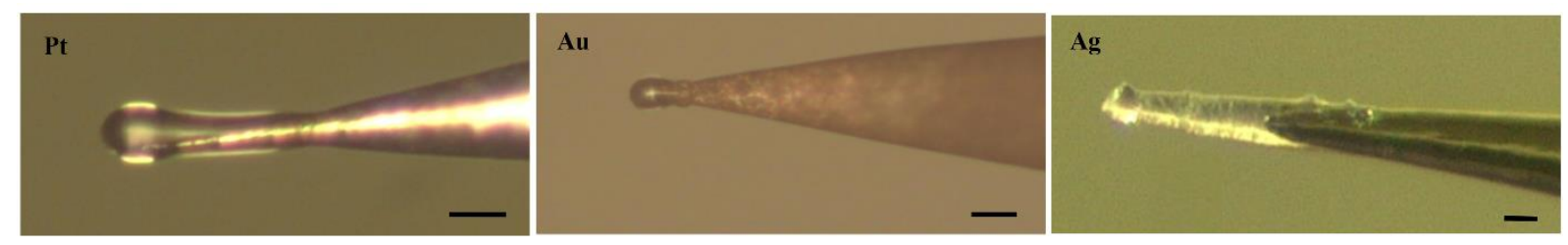

Fig. S2. Examples of sharpened hydrogel fabricated by "electrodeposition + pulling" on sharpened Pt, Au and Ag wires. Scale bar $25 \mu \mathrm{m}$.

\subsection{Experimental setup for local electrochemical modification of a 3D printed model}

The photo of the experimental setup for locally modifying a 3D printed model of Eiffel Tower is shown in Fig. S3. It should be noted that the model is placed in the same direction as printed, without any special adjustment in position angle. This demonstrates the applicability of hydrogel probes to any shape.

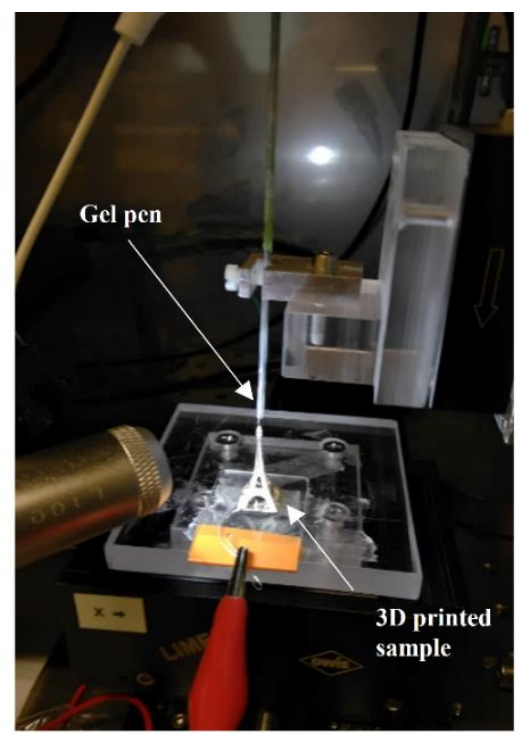

Fig. S3. Photo of the experimental setup for locally modifying the 3D printed Eiffel Tower model.

\section{Results and Discussion}

\subsection{Influence of holding parameters on the shape of the hydrogel}

Systematic experimental research has been carried out to understand the individual roles of each parameter in the gel formation during "electrodeposition + pulling". As the deposition is 
dominated by the pulling step, the pulling parameters have the highest impact on the shape of the deposit. Thus, their influence and tuning are discussed in detail in the main text. At the same time, it is also essential to initiate the electrodeposition of chitosan already at the holding step. The main purpose is to generate a "seed" that guides the further deposition under dynamic pulling. It can be tuned by holding parameters $L, E_{h}$ and $t_{h}$. Once the metal wire touches the deposition solution, the area of contact between the metal and the solution can be further increased via purposely immersing the wire by a distance $L$ after detecting the contact. The length of immersion $L$ affects the initial electrodeposition when holding the wire at $E_{h}$ for $t_{h}$ before pulling up. Fig. $\mathbf{S 4}$ reveals the effect of $L$ on the shape of gel deposition. At $L=1 \mu \mathrm{m}$, there is no deposition at the end of the tip even by applying $-1.3 \mathrm{~V}$ for a long time (300 s) (Fig. S4A). This is because the electrochemically generated $\mathrm{OH}^{-}$ions can easily diffuse away from the metal tip through hemispherical diffusion, making the local $\mathrm{pH}$ increase much more difficult than on planar electrodes. Similar phenomenon was observed when electrodepositing silica on micro-disk electrodes [5] and electrodepositing chitosan on cut wires [6], which could also explain the difficulty of electrodepositing chitosan at the tip of etched metal wires. Therefore, it is essential to immerse the metal wire deeper in the solution to initiate the electrodeposition of chitosan. Fig. S4B shows the gel deposited with $L=20 \mu \mathrm{m}$. Significant deposit is observed at the side of the immersed conical tip of the metal wire, and by adding a pulling step ("electrodeposition + pulling") the deposition could be guided in the direction parallel to the metal wire (Fig. S4C). Even though the shape of the hydrogel is significantly different from Fig. S4B, the area of contact between the gel and the metal cone is almost identical. This suggests that one may tune the "root" of gel deposit by the length of immersion $L$. If $L$ is too large, the deposited gel is very wide and blunt (Fig. S4D), which is unsuitable for spatially localized electrochemical measurements. Therefore, the choice of $L$ is a compromise to initiate the electrodeposition of chitosan on metal but not to have excess deposition at the side of the tip. In practice, we recommend setting $L$ in the range of $10-30 \mu \mathrm{m}$. 


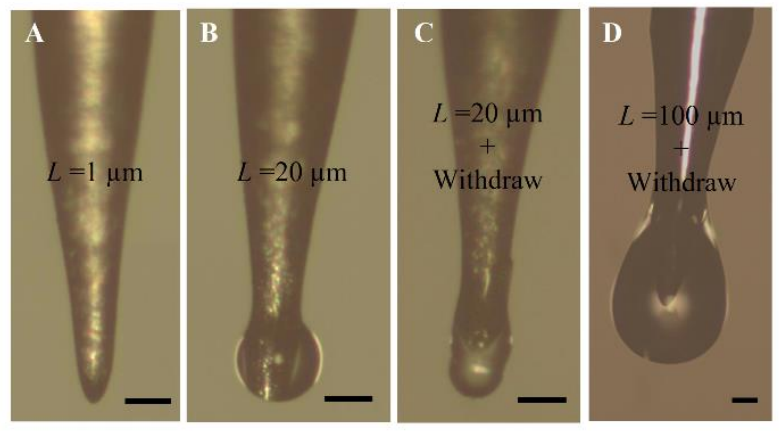

Fig. S4. Effect of the length of immersion $(L)$ on the initial deposition of the gel on an etched W wire. A- $L=1 \mu \mathrm{m}$, B- $L=20 \mu \mathrm{m}$, and after "electrodeposition + pulling" at C- $L=20 \mu \mathrm{m}$ and D- $L=100 \mu \mathrm{m}$. Conditions of deposition: $E_{a}=-0.7 \mathrm{~V} ; E_{h}=-0.9 \mathrm{~V} ; t_{h}=5 \mathrm{~s} ; v_{w l}=2 \mu \mathrm{m} \mathrm{s}^{-1} ; E_{w}=-1.2 \mathrm{~V}$. Scale bar: $25 \mu \mathrm{m}$.

After positioning the etched metal wire at an immersed distance $L$, the next step is to apply potential $E_{h}$ for $t_{h}$ while holding the wire. This holding step drives the initial deposition of chitosan forming "seed" that anchors further deposition in the later pulling step. Fig. S5 compares the hydrogel deposited with different $E_{h}$ and $t_{h}$, using the same conditions for the other steps of fabrication. It is clearly seen that gel fails to deposit on the wire when applying not sufficiently negative potential for short duration (Fig. S5A). Shifting $E_{h}$ more negative and prolonging $t_{h}$ would allow deposition at the tip, which continues in the pulling step forming a thin tubular shaped hydrogel (Fig. S5B). In contrary, holding the wire at very negative $E_{h}$ for even longer $t_{h}$ would yield excessive deposition at the root near the metal tip (Fig. S5C). The excessive deposition is not surprising and can be well explained by the $\mathrm{pH}$ gradient[7]. Nevertheless, it is interesting to see a "tail" that has similar width and length as in Fig. S5B, even though it appears twisted. This suggests that the shape of the main tubular part of hydrogel deposition is not significantly affected by the holding conditions. In short, the holding step should yield sufficient initial deposition to be pulled in the next step, however excessive deposition should also be avoided as it might affect the mechanical properties of the hydrogel (which might be the reason for the twisting). 

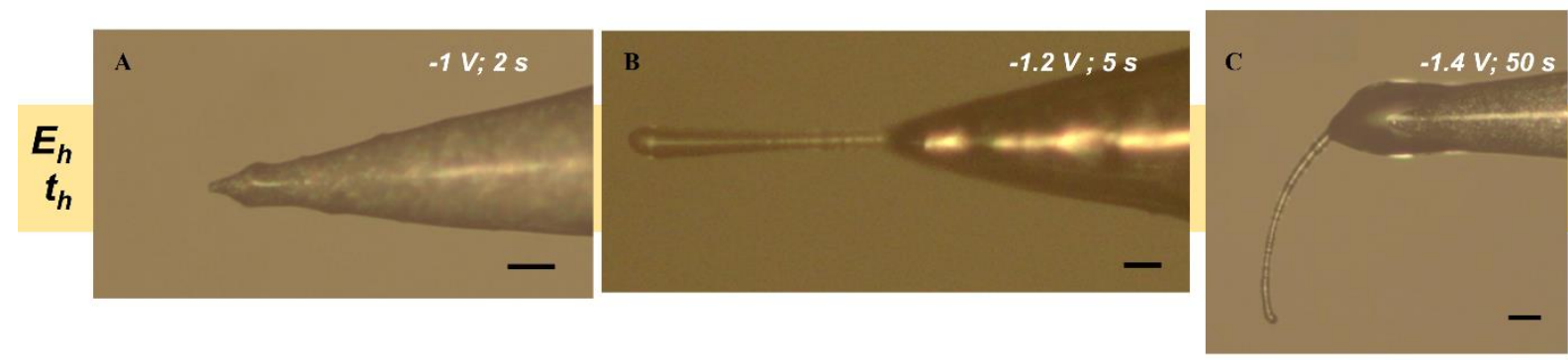

Fig. S5. Effect of $E_{h}$ and $t_{h}$ on the shape of the gel on etched W wire. Conditions of deposition: $E_{a}=-0.7$ $\mathrm{V} ; L=20 \mu \mathrm{m} ; E_{w}=-1.2 \mathrm{~V} ; v_{w l}=2 \mu \mathrm{m} \mathrm{s}^{-1}$. Values for $E_{h}$ and $t_{h}$ are indicated in the figures. Scale bar: 25 $\mu \mathrm{m}$.

In summary, the guideline for adjusting holding parameters is to have sufficient initial deposition of chitosan for guiding the further deposition in the later pulling step, but not to have excess deposition that makes the "root" of gel deposit too wide. Generally, $L$ is recommended to be in the range of $10-30 \mu \mathrm{m}$, and more deposition is obtained by applying more negative $E_{h}$ for longer $t_{h}$. At the holding step, the metal wire is positioned statically, so the deposition is similar as classical electrodeposition and the parameters can be relatively easily fixed. The holding parameters also have less influence on the shape of the gel deposit as compared with the pulling parameters.

\subsection{Reproducibility of "electrodeposition + pulling"}

Fig. S6 shows four hydrogels prepared with the same conditions from the same deposition solution. Generally, the geometry of the gel is reproducible and can be predicted quite well.
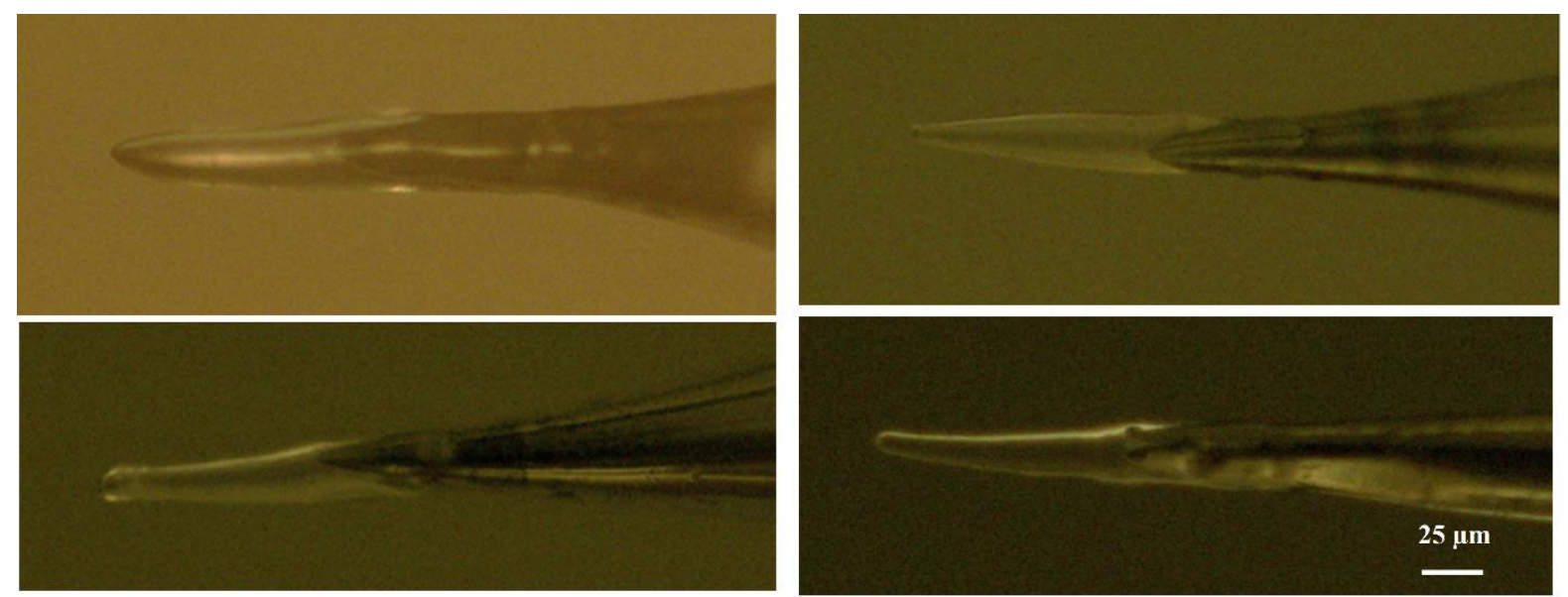

Fig. S6. Reproducibility of the "electrodeposition + pulling" in the hydrogel deposit applying the same parameters. Conditions of deposition: $E_{a}=-0.7 \mathrm{~V} ; L=30 \mu \mathrm{m} ; E_{h}=-0.9 \mathrm{~V} ; t_{h}=2 \mathrm{~s} ; E_{w}=-0.8 \mathrm{~V}, v_{w l}=1$ $\mu \mathrm{m} \mathrm{s}^{-1}$. 


\subsection{Local electrochemical writing of $\mathrm{AgCl}$}

Fig. S7 presents further analysis of the array shown in Fig. 5C $(R \sim 6 \mu \mathrm{m})$. EDX mapping confirms the chemical nature of the spots to be AgCl. From SEM images and height profile, it seems that the spots become highly homogeneous in shape and height, consisting of essentially $\mathrm{AgCl}$ particles as confirmed by EDX mapping (with the absence of oxygen signals).
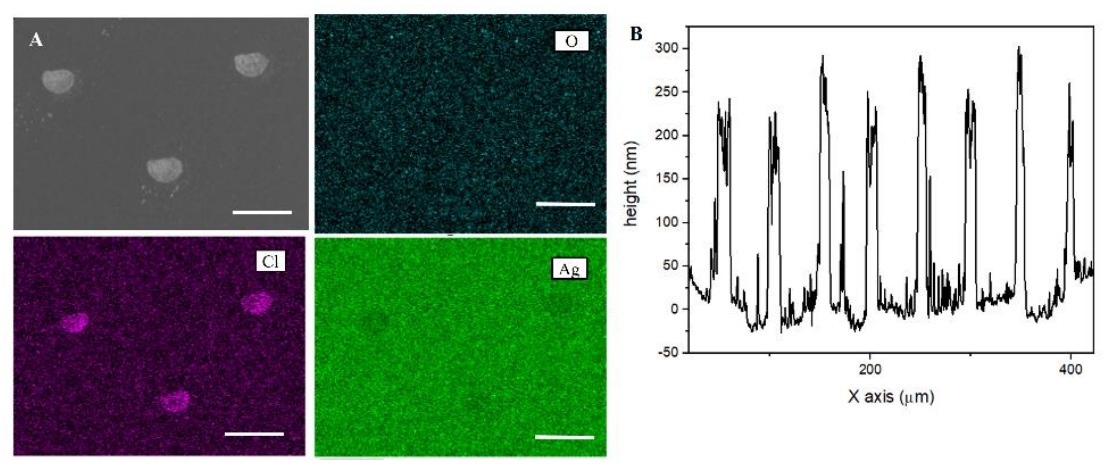

Fig. S7. A- SEM and EDX mapping of random $\mathrm{AgCl}$ spots. B- Height profile of a column of spots. Scale bar: $25 \mu \mathrm{m}$.

The resolution of local electrochemical writing is further challenged with a thinner hydrogel of high aspect ratio. Fig. S8 shows the complete array of 121 spots from where Fig. 5D $(R \sim 2$ $\mu \mathrm{m})$ is taken. Each spot was written by applying $E_{\text {Sample }}$ vs. $E_{g e l}=0.6 \mathrm{~V}$ for $\mathrm{t}=3 \mathrm{~s}$ at $z=0 \mu \mathrm{m}$. It is seen that the pattern generally appears as controlled. Nevertheless, some spots are significantly smaller than the others, and a few spots are either missing or laterally displaced. This is likely due to the lateral shift of the sharpened hydrogel tip, which affects the sense of contact by current feedback. It indicates that the hydrogel, as well as the instrumentation still need to be further improved for high resolution writing. 

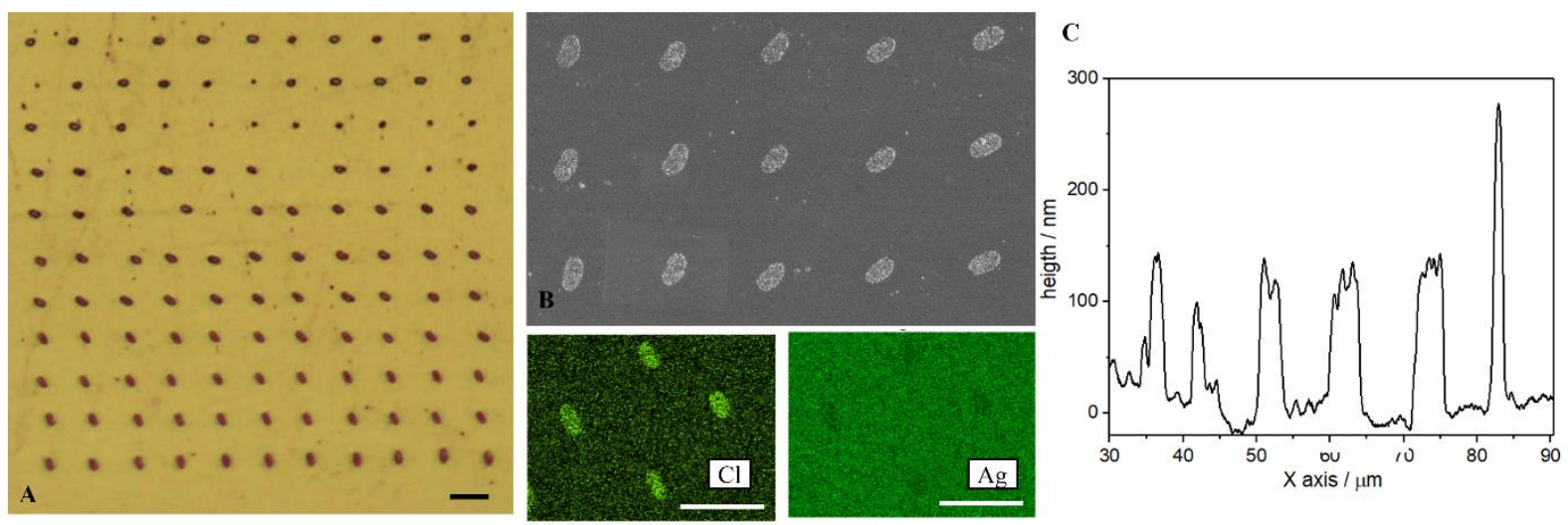

Fig. S8. A-Photo of an array of $\mathrm{AgCl}$ spots written with a high resolution hydrogel (Fig. S15B). B- SEM image and EDX map for $\mathrm{Ag}, \mathrm{Cl}$ and $\mathrm{O}$ of randomly selected spots. C- Height profile of the deposited $\mathrm{AgCl}$ spots. Scale bars: $10 \mu \mathrm{m}$.

Fig. S9A shows the complete array from where Fig. 5C $(R \sim 64 \mu \mathrm{m})$ is extracted. The flexible resolution feature of hydrogel probes is demonstrated by stretching and pressing the hydrogel after touching the surface. Here, each column is generated with the same voltage and time ( $E_{\text {Sample }} \mathrm{vs} . E_{\text {gel }}=0.6 \mathrm{~V} ; \mathrm{t}=8 \mathrm{~s}$ ) but at different $z$. It is clearly seen that the diameter of the spots increases as the hydrogel is pressed against the sample ( $z$ shifts from -3 to $+15 \mu \mathrm{m}$ ). The diameter as a function of $z$ is almost linear in the range of $z=0$ to $+15 \mu \mathrm{m}$. Moreover, the patterns at $z=0$ and $+5 \mu \mathrm{m}$ are repeated after writing at $z=+15 \mu \mathrm{m}$. The spots are almost the same as the second and third rows, indicating that the pressing of gel up to $+15 \mu \mathrm{m}$ is elastic and the shape of hydrogel is well restored after detaching. This way, the area of contact between the hydrogel and the surface can be tuned by $z$ position (in an almost linear fashion for $z>0$, as shown in Fig. S9B). It offers flexible resolution of electrochemical writing with the same gel probe[8].
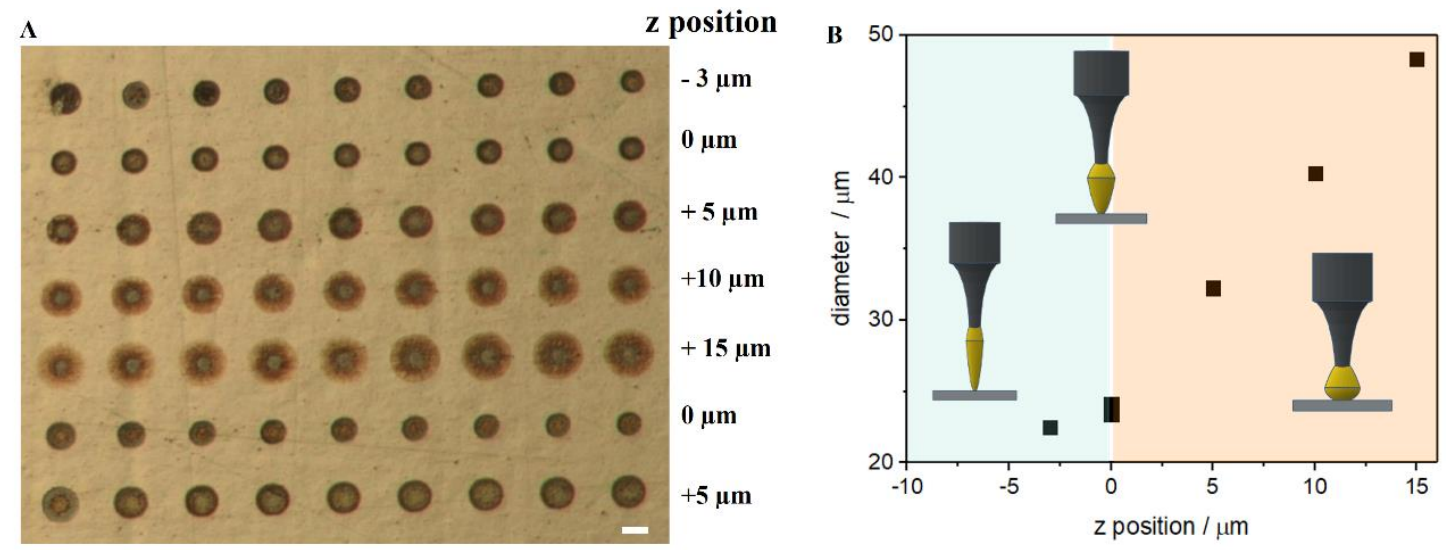
Fig. S9. A- Photo of an array of $\mathrm{AgCl}$ spots patterned with tunable resolution by controlling $z$. B- Diameter of spots $v s$. $z$ position curve. Scale bars: $25 \mu \mathrm{m}$.

\subsection{Local electrochemical etching of Ag}

Local modification of $\mathrm{Ag}$ surfaces is further tested for etching $\mathrm{Ag}$-coated ITO using $\mathrm{NO}_{3}^{-}-$ containing gel probes. Same as oxidizing $\mathrm{Ag}$ with $\mathrm{Cl}^{-}$-containing hydrogel, the first step is to approach the probe to be in contact with Ag surface. Then, the hydrogel is held at $z=0$ where consecutive scans of cyclic voltammetry is measured (Fig. S10A). It shows that the oxidation peak current gradually decreases, which indicates the dissolution of Ag. After a few scans, the peak becomes almost invisible, suggesting that only the ITO substrate is exposed. Thus, a constant potential of $E_{\text {Sample }} v s . E_{g e l}=1 \mathrm{~V}$ is chosen for locally etching Ag. The bias is applied for $20 \mathrm{~s}$ at $z=-2 \mu \mathrm{m}$ for each spot, yielding a series of spots shown in Fig. S10B. The removal of Ag is confirmed by profilometry and EDX (Fig. S10C-D). The size (ca. $4 \mu \mathrm{m})$ and shape of the etched holes are quite reproducible.

More examples of local electrochemical etching by hydrogels are shown in Fig. S11, demonstrating that the process works at very different resolution of $c a .25$ and $2 \mu \mathrm{m}$. These results further confirm the feasibility of using hydrogel probes for local electrochemical etching, yet etching more spots with the same hydrogel probe remains challenging at this stage due to the contamination of the gel which will be discussed in Section 3.
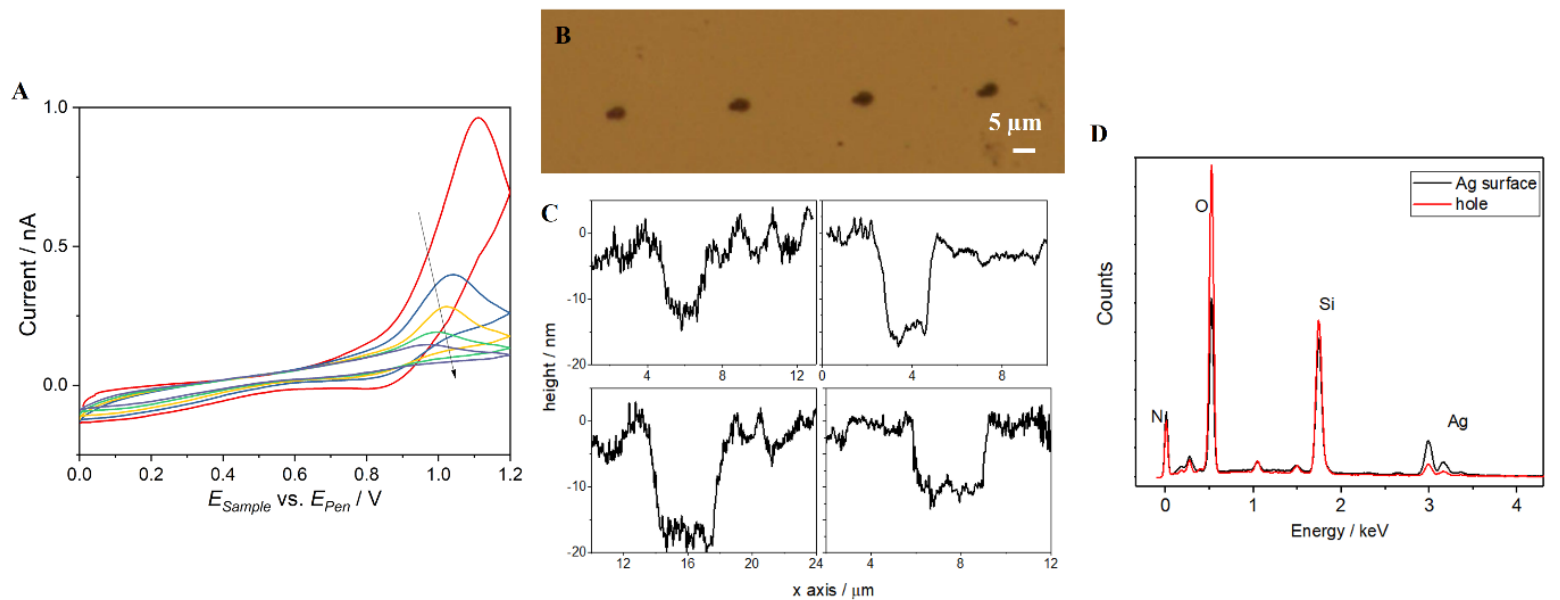

Fig. S10. A- Consecutive CV scans of Ag-coated ITO using a hydrogel containing $\mathrm{NO}_{3}^{-}$. B- Photo of an array of etched holes on Ag-coated ITO. C- Depth profiles of the patterned holes. D- EDX spectra of the etched sample. 

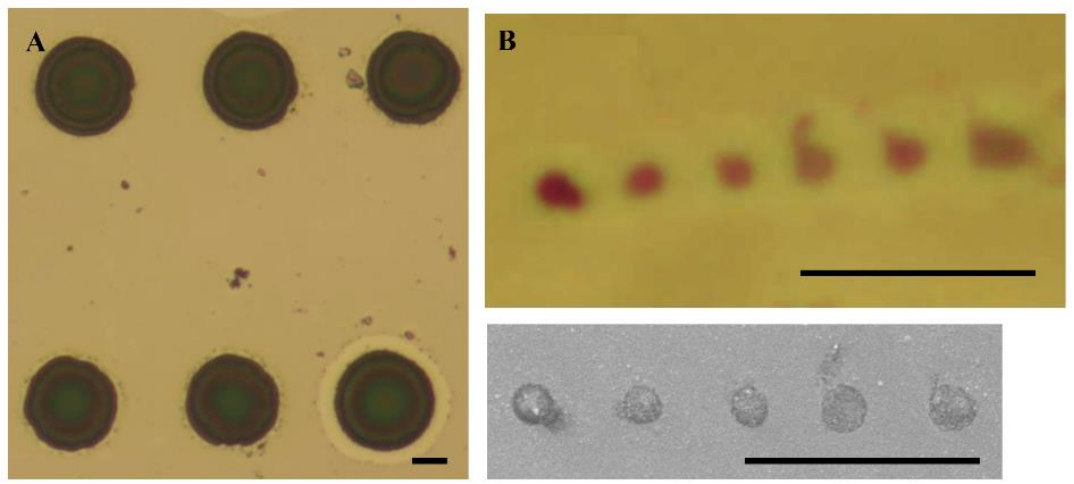

Fig. S11. A and B- Photos and SEM image of etched holes on Ag-coated ITO with low and high resolution. Scale bars: $10 \mu \mathrm{m}$.

\subsection{Effect of sample tilt on the electrochemical writing}

One major advantage of the hydrogel is the soft contact with the sample, which allows local electrochemical measurements on complex shaped surfaces. To mimic the complex shape, we purposely tilt a flat sample and examine its effect on the electrochemical writing of $\mathrm{AgCl}$. Fig. S12 shows the spots of $\mathrm{AgCl}$ written on the Ag plate positioned with different tilt angle. All the spots are written at contact position $(z=0 \mu \mathrm{m})$ with $E_{\text {Sample }} v s . E_{g e l}=1.2 \mathrm{~V}$ for $20 \mathrm{~s}$. Between spots, the hydrogel probe is laterally moved by $50 \mu \mathrm{m}$ either in $x$ or $y$ axis. Table $\mathbf{S 2}$ summarizes the average diameter of the spots and the average distance between adjacent spots in a row. It is seen that the spots written with $\theta=60^{\circ}$ and $45^{\circ}$ have the same diameter as those written at classical vertical position $\left(\theta=90^{\circ}\right)$. The distance between adjacent spots also fits well the calculated values $\left(d_{y}=50 \mu \mathrm{m}, d_{x}=50 \mu \mathrm{m} / \sin \theta\right)$. At $\theta=30^{\circ}$, the diameter of spots significantly reduces by $c a$. $50 \%$, whereas the line starts to be less straight. The $d_{x}$ is also smaller than calculated. We further challenge the writing at $\theta=10^{\circ}$. Spots with even smaller diameter can still be clearly seen, but the shape becomes irregular and the distance between spots cannot be accurately controlled.

The results suggest that the hydrogel probes fabricated by "electrodeposition + pulling" can reliably write on surfaces with tilt up to $45^{\circ}$, with no significant change in the resolution. It is also possible to write on steeper planes up to $\theta=30^{\circ}$ with acceptable quality. Further reducing $\theta$ to $10^{\circ}$ would bring difficulties in control and therefore is less reliable. The exact limit of 
sample tilt may also depend on the shape of the gel deposit and even the etching of metal wire. Nevertheless, this is generally sufficient as the approaching direction of the hydrogel can also be rotated in practice (for example, flip the hydrogel probe by $90^{\circ}$ and approach to the sample horizontally). The results suggest that the hydrogel probe is not sensitive to the positioning of the sample and pave the way for locally modifying complex shaped surfaces such as 3D printed structures shown in the main text.

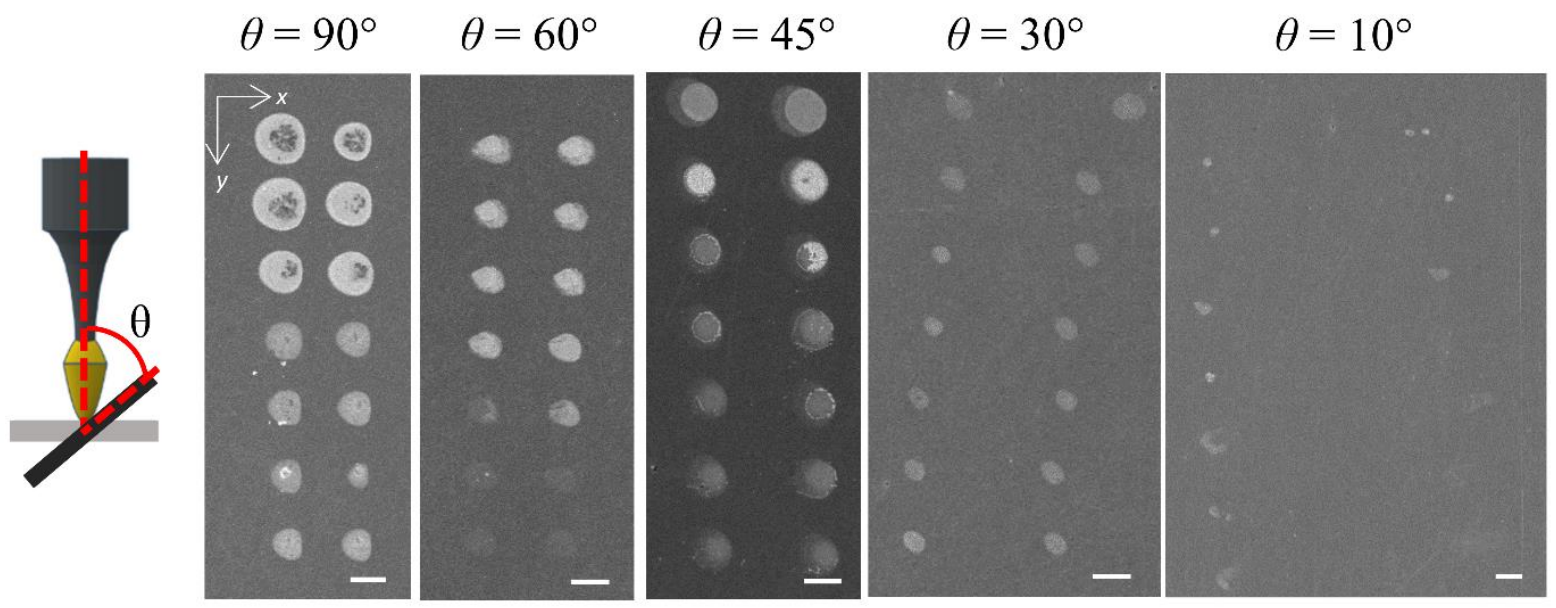

Fig. S12. Scheme of experimental setup and photos of $\mathrm{AgCl}$ spots written at different angles on $\mathrm{Ag}$ foil. Scale bar: $25 \mu \mathrm{m}$.

Table S2. Effect of tilting on the geometry of patterns shown in Fig. S12.

\begin{tabular}{cccc}
\hline$\theta$ & $\begin{array}{c}\text { Average spot } \\
\text { diameter } \\
(\mu \mathrm{m})\end{array}$ & $\begin{array}{c}\text { Average distance } \\
\text { between two adjacent } \\
\text { spots in a row } d_{x}(\mu \mathrm{m})\end{array}$ & $d_{x}$ calculated $(\mu \mathrm{m})$ \\
\hline $90^{\circ}$ & 20.97 & 50 & 50 \\
\hline $60^{\circ}$ & 20.69 & 56.7 & 57.7 \\
$45^{\circ}$ & 20.15 & 68.13 & 70.71 \\
$30^{\circ}$ & 13.64 & 95.07 & 100 \\
$10^{\circ}$ & 8.79 & 241.35 & 287.9 \\
\hline
\end{tabular}

\subsection{Local electrochemical modification of a 3D printed Eiffel Tower}

Complementary to the example given in Fig. 6B, Fig. S13 presents several points of $\mathrm{AgCl}$ that are written at the sharply curved edges (patterns B and C) as well as the side wall details (pattern 
D, shown in Fig. 6B) of the 3D printed Eiffel Tower by applying $E_{\text {Sample }} v s$. $E_{\text {gel }}=1.2 \mathrm{~V}$ (as marked in Fig. S13A). Pattern B (Fig. S13B) shows a dash line of $c a .10 \mu \mathrm{m}$ width made at $z=$ $0 \mu \mathrm{m}(t=50 \mathrm{~s}$ for each point $)$. Pattern $\mathrm{C}$ shows spots of $c a .25 \mu \mathrm{m}$ diameter assembled in a long line of $3 \mathrm{~mm}$ (Fig. S13C, showing only part of the line) crossing the edge (marked with dash line in Fig. S13C). The spots are made at $z=+5 \mu \mathrm{m}$ by applying the same potential for $10 \mathrm{~s}$ for each spot. It is seen that the precision is maintained even by significantly changing the angle of the surface from one plane to another.

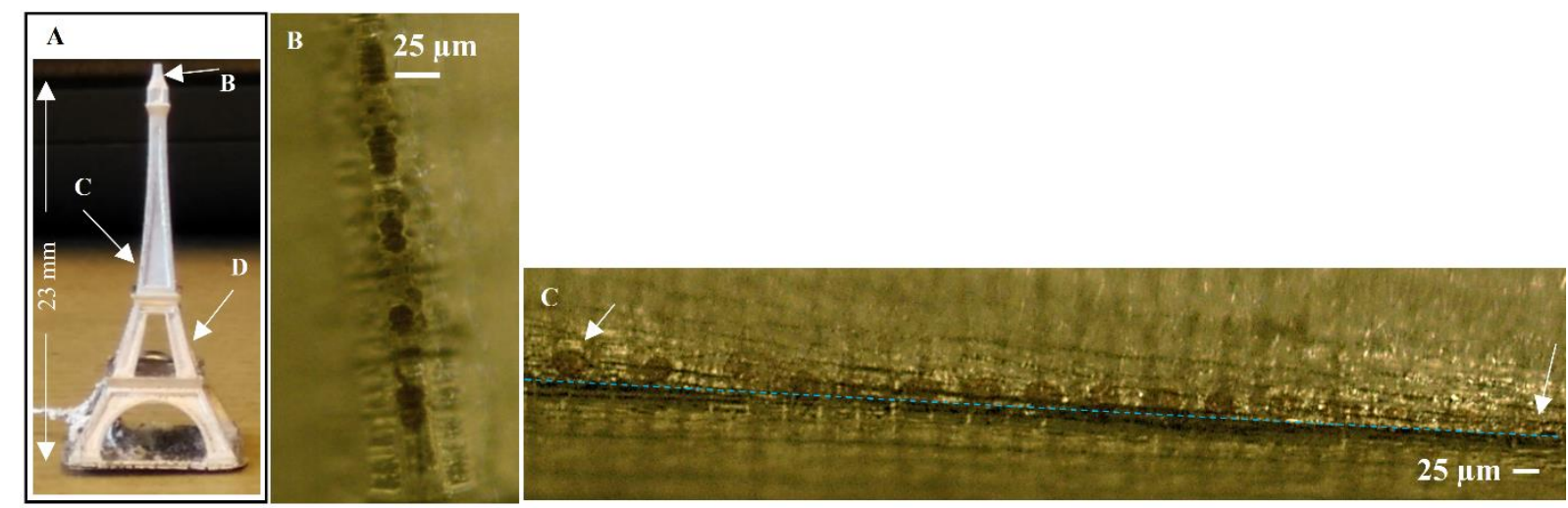

Fig. S13. A- 3D printed Eiffel Tower model coated with Ag showing the edges (B and C) and steps (D) where patterns of $\mathrm{AgCl}$ are made. $\mathbf{B}$ and $\mathbf{C}$ - photos of patterns of $\mathrm{AgCl}$ made by the hydrogel probe shown in Fig. S14.

\section{Technical aspects: printing speed and stability of hydrogel probes}

The feasibility of hydrogel probes for locally modifying or etching (complex-shaped) surfaces by electrochemistry is well demonstrated. As all other printing techniques, a major technical aspect is the speed. This is determined by the time of approach, hold and withdraw. The approaching and withdrawing time depend on the vertical traveling distance and therefore depend on the geometry of the sample. One could estimate that for a traveling distance of 50 $\mu \mathrm{m}$, it takes $5 \mathrm{~s}$ when approaching the piezo at $10 \mu \mathrm{m} \mathrm{s}^{-1}$. This would provide a positioning accuracy of $10 \mathrm{~nm}$ when using a feedback loop at $1 \mathrm{kHz}$. The hydrogel probe can be withdrawn at the maximum speed of piezo if the retract curve is not recorded, and thus the time is negligible as compared to approaching. Nevertheless, it should be noted that the hold time depends on the kinetics of the local electrochemical reactions. This cannot be optimized by the engineering of the instrument and has a theoretical limit by Faraday's Law for metal deposition/dissolution. 
Another practical concern of the hydrogel probe is the stability. This includes two aspects: the mechanical stability and the chemical stability. The former can be examined by comparing the shape of the gel before and after experiments. The latter is more difficult to be determined but a rough estimation can be derived from Faraday's Law, as discussed later.

Fig. S14 summarizes the photos of hydrogel probes before and after local modification of surfaces in the present work. In general, there is not major change in the shape of the gel after being used (multiple pressing and stretching against/from the surface) for long time (hours). An exception is the hydrogel probe shown in Fig. S14B, where the thin and long tubular gel is clearly bent after writing 121 points at high resolution of $c a .1-2 \mu \mathrm{m}$. It can explain the lateral drift in the shape of the written spots (Fig. S8A). However, the contact area did not change significantly despite the bending. The mechanical stability of the hydrogel probes shall be further improved by optimizing the gel material in future.

To analyze the chemical stability (depletion/accumulation) of the hydrogel probes, a rough estimation can be derived from Faraday's Law:

$c=\frac{Q_{F} n_{\text {spot }} f}{n F V_{g e l}}$

where $c$ is the concentration of species that are electrochemically generated or consumed from the gel, $Q_{F}$ refers to the Faradaic charge at each spot, $n_{s p o t}$ refers to the number of spots, $f$ refers to the Faradaic efficiency, $n$ is the number of electron involved in reaction, $F$ is the Faraday Constant, and $V_{g e l}$ represents the volume of the gel.

$Q_{F}$ can be further expressed as:

$Q_{F}=A \int_{0}^{t} i_{F} d t$

where $A$ is the contact area between the gel and the sample, $i_{F}$ is the Faradaic current density. By applying an approximation that the gel is cylindrical, and its diameter is proportional to the contact area by a factor of $k$, then,

$V_{g e l}=k A l_{g e l}$ 
where $l_{g e l}$ is the length of the gel. Substitute Equation 3 by Equation 4 and 5:

$c=\frac{n_{\text {spot }} f}{n F k l_{\text {gel }}} \int_{0}^{t} i_{F} d t$

From Equation 6, it can be seen that with the same Faradaic current density, $c$ is higher if the gel is shorter. In practice, to prevent the uncontrollable lateral shift of the hydrogel it is recommended to use shorter gel for patterning at high resolution (Fig. S8), and this is why we employed a second pulling step to "cut" the gel. Then inevitably, the problem of accumulation/depletion becomes more dominant. Fig. S14H shows a clear example of accumulation of reaction products: the gel turns dark after etching a few spots. We suspect that this is related to the saturation of oxidation products $\left(\mathrm{AgNO}_{3}\right)$, which could be avoided by refreshing the hydrogel via soaking electrolyte in future. In this work we did not observe significant depletion of $\mathrm{Cl}^{-}$ions in the gel while locally modifying $\mathrm{Ag}$ to form $\mathrm{AgCl}$. It should also be noted that considering the ease of "electrodeposition + pulling" process, the hydrogel can also be regenerated by removing the gel and redeposit, like for Fig. 4 . 


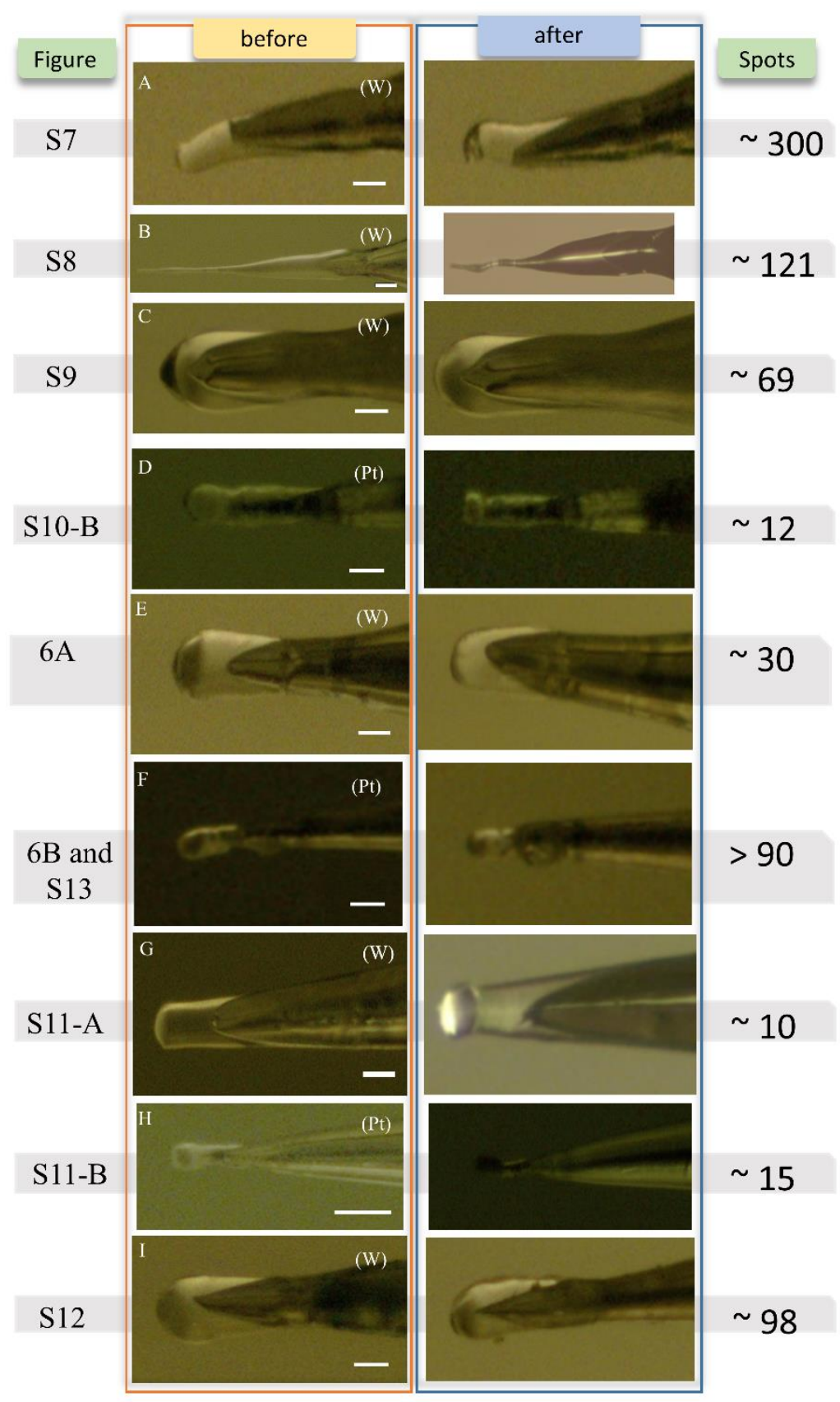

Fig. S14. Summary of the hydrogels used for electrochemical pattering in this work. Scale bars: $25 \mu \mathrm{m}$. 4. Videos

\subsection{Video S1: Electrodeposition + Pulling}

Video S1 shows the process of "Electrodeposition + Pulling" fabrication of sharp chitosanbased hydrogel applied to a sharpened $\mathrm{W}$ wire. The three steps of the protocol (approach, hold and pulling) can be seen in the clip. The stretching of the meniscus during the pulling leads to the final tubular shape of the hydrogel as seen in the video. The video is recorded at real speed, 
and in this case the whole process takes less than two minutes. But it may take longer or shorter time depending on the conditions and the desired shape of the hydrogel.

\subsection{Video S2: Local modification of 3D printed Eiffel Tower model}

Video S2 is recorded while writing at the edge of the top of the 3D printed Eiffel Tower. As explained in the main text, the patterns are made using the hopping sequence (approach, hold, retract and then move to next point). In this case, the program executes a linear pattern of 8 spots holding the hydrogel probe at $1 \mathrm{~V}$ for $5 \mathrm{~s}$ at each spot $(z=0)$. The speed during approach and retraction is 20 and $110 \mu \mathrm{m} \mathrm{s}^{-1}$, respectively. It should be noted that the conditions are different from that used for Fig. 6B in the main text. They are not suitable for obtaining good patterns, but the video illustrates the process.

\subsection{Video S3: Writing at the step edge of the 3D printed Eiffel Tower}

Video $\mathbf{S 3}$ shows a pattern of $\mathrm{AgCl}$ spots at the edge of printed Eiffel Tower model, which is presented in Fig. 6B of the main text. Due to the complex shape of the surface, it is impossible to have all the spots in the same optical focus, so the image of Fig. $\mathbf{6 B}$ is acquired with only the right bottom part (4 spots at Row 1) in focus and the rest blurred. To better illustrate the quality of pattern on this complex shape, Video $\mathbf{S 3}$ shows the sample under optical microscope when the focus is gradually changed from Row 1 to the channel and then to Row 2.

\section{References}

[1] M. Lopes, T. Toury, M.L. De La Chapelle, F. Bonaccorso, P. Giuseppe Gucciardi, Fast and reliable fabrication of gold tips with sub-50 $\mathrm{nm}$ radius of curvature for tip-enhanced Raman spectroscopy, Rev. Sci. Instrum. 84 (2013) 073702-1-073702-8. https://doi.org/10.1063/1.4812365.

[2] L. Libioulle, Y. Houbion, J.M. Gilles, Very sharp platinum tips for scanning tunneling microscopy, Rev. Sci. Instrum. 66 (1995) 97-100. https://doi.org/10.1063/1.1146153.

[3] C. Zhang, B. Gao, L.G. Chen, Q.S. Meng, H. Yang, R. Zhang, X. Tao, H.Y. Gao, Y. Liao, Z.C. Dong, Fabrication of silver tips for scanning tunneling microscope induced luminescence, Rev. Sci. Instrum. 82 (2011) 083101-1-083101-5. https://doi.org/10.1063/1.3617456.

[4] G. Tahmasebipour, Y. Hojjat, V. Ahmadi, A. Abdullah, Effect of fabrication process parameters on the apex-radius of STM tungsten nanotip, Scanning. 31 (2009) 65-74. https://doi.org/10.1002/sca.20142.

[5] G. Herzog, N.A. Vodolazkaya, A. Walcarius, Platinum ultramicroelectrodes modified 
with electrogenerated surfactant-templated mesoporous organosilica films: Effect of film formation conditions on its performance in preconcentration electroanalysis, Electroanalysis. 25 (2013) 2595-2603. https://doi.org/10.1002/elan.201300415.

[6] K. Yan, F. Ding, W.E. Bentley, H. Deng, Y. Du, G.F. Payne, X.-W. Shi, Coding for hydrogel organization through signal guided self-assembly, Soft Matter. 10 (2014) 465469. https://doi.org/10.1039/c3sm52405a.

[7] Y. Liu, A. Holzinger, P. Knittel, L. Poltorak, A. Gamero-Quijano, W.D.A. Rickard, A. Walcarius, G. Herzog, C. Kranz, D.W.M. Arrigan, Visualization of Diffusion within Nanoarrays, $\quad$ Anal. $\quad$ Chem. 88 (2016) 6689-6695. https://doi.org/10.1021/acs.analchem.6b00513.

[8] F. Huo, Z. Zheng, G. Zheng, L.R. Giam, H. Zhang, C.A. Mirkin, Polymer pen lithography, Science. 321 (2008) 1658-1660. https://doi.org/10.1126/science.1162193. 